\title{
DYNAMIC ANALYSIS OF A MULTIVARIATE REWARD PROCESS DEFINED ON THE UMCP WITH APPLICATION TO OPTIMAL PREVENTIVE MAINTENANCE POLICY PROBLEMS IN MANUFACTURING
}

\author{
Jia-Ping Huang \\ Department of Econometrics and OR, FEWEB \\ VU University Amsterdam \\ De Boelelaan 1105, 1081 HV Amsterdam, The Netherlands \\ E-mail: j.huang@vu.nl \\ Ushio Sumita \\ Graduate School of Systems and Information Engineering \\ University of Tsukuba \\ 1-1-1 Tennoudai, Tsukuba, Ibaraki 305-8573, Japan \\ E-mail: sumita@sk.tsukuba.ac.jp
}

\begin{abstract}
The unified multivariate counting process (UMCP), previously studied by the same authors, enables one to describe most of the existing counting processes in terms of its components, thereby providing a comprehensive view for such processes often defined separately and differently. The purpose of this paper is to study a multivariate reward process defined on the UMCP. By examining the probabilistic flow in its state space, various transform results are obtained. The asymptotic behavior, as $t \rightarrow \infty$, of the expected univariate reward process in a form of a product of components of the multivariate reward process is studied. As an application, a manufacturing system is considered, where the cumulative profit given a preventive maintenance policy is described as a univariate reward process defined on the UMCP. The optimal preventive maintenance policy is derived numerically by maximizing the cumulative profit over the time interval $[0, T]$.
\end{abstract}

Keywords unified multivariate counting process; multivariate reward process; transform results; asymptotic analysis; preventive maintenance policy

\section{Introduction}

The history of counting processes can be traced back to 1950's stemmed from introduction of Poisson processes, see e.g. Feller [4] . Since then, many different counting processes have been introduced in response to many different application needs, including non-homogeneous Poisson processes, renewal processes (see, e.g. Ross [23]), Markov-modulated Poisson processes (Heffes and Lucantoni 
[6]), Markov renewal processes (Pyke [20, 21], Keilson [8, 9], Keilson and Wishart [10, 11]), Markovian arrival processes (Lucantoni, Meier-Hellstern and Neuts [13], Lucantoni [12]) and other agedependent counting processes (Masuda and Sumita [16], Sumita and Shanthikumar [28]), to name a few. Such counting processes have been introduced separately with different motivations. Accordingly, they seem to be quite different on the surface.

In order to provide a comprehensive view over such different counting processes, a unified multivariate counting process (UMCP) is introduced in Sumita and Huang [26], where the UMCP enables one to describe most of the existing counting processes in terms of its components. More specifically, a stochastic system is considered, where a semi-Markov process $J(t)$ defined on $\mathscr{J}=\{0,1, \cdots, J\}$ constitutes the core process with the counting process $N_{i j}(t)$ denoting the number of entries of $J(t)$ from state $i \in \mathscr{J}$ to state $j \in \mathscr{J}$. Also incorporated is the counting process $M_{i}(t)$ recording the number of arrivals in state $i \in \mathscr{J}$, where such arrivals are generated by the non-homogeneous Poisson process governed by the intensity function $\lambda_{i}(x)$ depending on the current dwell time $x$ of the semi-Markov process in state $J(t)=i \in \mathscr{J}$ at time $t$.

As an application of the UMCP, a manufacturing system with certain optimal preventive maintenance policy is considered in [26], where the system degrades through multiple stages described by the state of the semi-Markov process $J(t)$ on $\mathscr{J}=\{0, \cdots, J\}$. Here, state 0 is the perfect state, and the system degrades gradually from 0 to $J-1$ with default rate increasing along this direction. State $J$ corresponds to the overhaul for the preventive maintenance, where the system is completely stopped but would be brought back to the perfect state upon completion. The overhaul time would become stochastically longer as $J$ increases, that is, as the preventive maintenance is deferred. The total cost associated with the preventive maintenance policy $J$ is then given by the counting process $N_{J-1, J}(t)$ with $\psi_{m} \times N_{J-1, J}(t)$ representing the overhaul cost and $M_{i}(t)$ with $\psi_{d} \times M_{i}(t)$ generating the scrap cost. However, the model cannot cope with a more sophisticated revenue and cost structure, where revenues and costs may be generated in continuous time depending on the state of $J(t)$, as well as at the times of jumps of $N_{i j}(t)$ and $M_{i}(t)$ with random increments.

In order to overcome this difficulty and expand the applicability of the UMCP, this paper introduces a multivariate reward process $\underline{Z}(t)$ defined on the UMCP. By examining the probabilistic flow in the state space of $\underline{Z}(t)$, various transform results are obtained. The asymptotic behavior, as $t \rightarrow \infty$, of the expected univariate reward process in a form of a product of components of the multivariate reward process is studied. As an application, the manufacturing system considered above is revisited, where the cumulative profit given a preventive maintenance policy is described as a univariate reward process defined on the UMCP with random jumps. The optimal preventive maintenance policy is derived numerically by maximizing the cumulative profit over the time interval $[0, T]$.

The study of reward processes can be traced back to the 1950's represented by the original paper by Smith [24]. Subsequently, many papers have been published, including Jewell [7], McLean and Neuts [18], and Pyke and Schaufele [22], to name only a few. The reader is referred to two excellent survey papers by Çınlar [2,3]. Of particular interest to this paper are the reward processes defined on a semi-Markov process and the associated counting processes studied by Sumita and Masuda [27], Masuda and Sumita [17] and Masuda [15]. More recently, Stefanov [25] provides some interesting transform results, linking reward functions accumulated in a deterministic time interval with those accumulated within first passage times of the underlying Markov chain or the semi-Markov process.

The optimal preventive maintenance problem has been an important branch of research in both stochastic modeling and reliability theory. An early approach, represented by Barlow and Hunte [1], Flehinger [5] and Malik [14] among others, was based on renewal theory where the optimal policy would be determined so as to minimize the cost or the downtime due to repair. Since then, the problem 
has been analyzed from various angles. For capturing the overview of such developments, the reader is referred to three excellent survey papers by Pierskalla and Voelker [19], Valdez-Flores and Feldman [29] and Wang [30]. The unique feature of the optimal preventive maintenance problem discussed in this paper can be found in that the optimal maintenance policy is to maximize the expected profit over a planning period, where the expected profit consists of three parts: continuous earnings during uptimes, continuous costs during downtimes and a random fixed cost incurred whenever a maintenance activity takes place. In addition, two performance measures are considered and compared so as to demonstrate that the optimal policy for maximizing the mean time between failures could be different from that for maximizing the system availability.

In what follows, we succinctly summarize Sumita and Masuda [27], Masuda and Sumita [17] and Masuda [15]. Let $Z(t)$ be a reward process associated with a semi-Markov process $J(t)$ on $\mathscr{J}=\{0, \cdots, J\}$, where $Z(t)$ is characterized by a state dependent reward rate function $\rho: \mathscr{J} \rightarrow \mathbb{R}$. Formally, $Z(t)$ can be written as

$$
Z(t)=\int_{0}^{t} \rho(J(\tau)) d \tau .
$$

Let $X(t)$ be the age process of $J(t)$ describing the elapsed time up to time $t$ since the last transition into the current state. By analyzing the trivariate process $[J(t), X(t), Z(t)]$, various transform results have been obtained in [27], yielding the asymptotic expansions of the first two moments of $Z(t)$ as $t \rightarrow \infty$. Also analyzed are the reward accumulated during a first passage time of $J(t)$ and the first passage time of $Z(t)$ itself. Masuda and Sumita [17] extend the model in [27] to a multivariate setting. More specifically, a multivariate reward process $\underline{Z}(t)=\left[Z_{1}(t), Z_{2}(t), \cdots, Z_{K}(T)\right]^{\top}$ is considered, where

$$
\underline{Z}(t)=\int_{0}^{t} \underline{\rho}(J(\tau)) d \tau
$$

possibly with $Z_{k}(t), k=1,2, \cdots, K$, depending on each other. In Masuda [15], this process is further extended by incorporating random jumps at transition epochs of the underlying semi-Markov process $J(t)$.

The purpose of this paper is to introduce a multivariate reward process $\underline{Z}(t)=\left[Z_{1}(t), Z_{2}(t), \cdots\right.$, $\left.Z_{K}(T)\right]^{\top}$ defined on the UMCP, possibly with random jumps at the times of the transitions of the UMCP. Since the UMCP unifies various counting processes, this multivariate reward process enables one to treat all of the above reward processes as special cases. Furthermore, some new reward processes can also be introduced. As an application, the optimal preventive maintenance policy problem discussed in Sumita and Huang [26] will be revisited, where the multivariate reward process allows one to introduce revenues and costs generated in continuous time as well as random increments of the cost at jump epochs of the UMCP. By applying the asymptotic expansion of $\mathrm{E}\left[\prod_{k=1}^{K} Z_{k}(t)\right]$, numerical examples are provided for demonstrating how the optimal maintenance policy could be obtained in this new context.

Throughout the paper, vectors and matrices are underlined and double underlined respectively, e.g. $\underline{u}$ and $\underline{v}$. The vector of having all components equal to 1 is denoted by $\underline{1}$. Furthermore, $\underline{1}_{i}$ is the vector having all components equal to 0 except that the $i$-th component is 1 . Similarly, $\underline{1}_{i j}$ is the matrix having all components equal to 0 except that the $(i, j)$ component is 1 . The indicator function $\delta_{\{\text {Statement }\}}$ takes the value of 1 if Statement holds true and 0 otherwise. The limit to 0 from above is denoted by $0+$.

The structure of this paper is as follows. In Section 2, the UMCP is formally introduced and the multivariate reward process $\underline{Z}(t)$ defined on the UMCP is described in detail. Dynamic analysis of 
the multivariate stochastic system involving the UMCP and $\underline{Z}(t)$ is provided in Section 3, and various transform results are obtained. Based on these transform results, Section 4 analyzes the asymptotic behavior of $\mathrm{E}\left[\prod_{k=1}^{K} Z_{k}(t)\right]$ as $t \rightarrow \infty$. The optimal preventive maintenance policy problem is discussed in Section 5, and numerical examples are presented in Section 6.

\section{Model Description}

Let $\{J(t): t \geq 0\}$ be a semi-Markov process on $\mathscr{J}=\{0, \cdots, J\}$, and define the age process $X(t)$ as the elapsed time until time $t$ since the last transition of $J(t)$ into the current state. Two types of multivariate counting processes are considered, where a matrix counting process $\underline{\underline{N}}(t)=\left[N_{i j}(t)\right]$ describes the number of transitions of $J(t)$ from $i \in \mathscr{J}$ to $j \in \mathscr{J}$ in $[0, t]$, and a vector counting process $\underline{M}(t)=\left[M_{0}(t), \cdots, M_{J}(t)\right]^{\top}$ represents the number of arrivals of certain items in state $i \in \mathscr{J}$ in $[0, t]$. Given that $J(t)=i$ and $X(t)=x$ at time $t$, it is assumed that the counting process $M_{i}(t)$ is a non-homogeneous Poisson process governed by the intensity function $\lambda_{i}(x)$. The multivariate stochastic process $[\underline{M}(t), \underline{\underline{N}}(t)]$ enables one to describe a variety of counting processes in a unified manner, e.g. $N_{i}(t) \stackrel{\text { def }}{=} \sum_{\ell \in \mathscr{J}} N_{\ell i}(t)$ denotes the number of entries of $J(t)$ into state $i$ by time $t$, and $\sum_{i \in \mathscr{A}} M_{i}(t)$ with $\mathscr{A} \subset \mathscr{J}$ may describe the number of defects generated in states $\mathscr{A}$. Indeed, it has been shown in Sumita and Huang [26] that many known counting processes can be expressed in terms of $[\underline{M}(t), \underline{\underline{N}}(t)]$. Because of this, $[\underline{M}(t), \underline{\underline{N}}(t)]$ is called the unified multivariate counting process (UMCP) and is analyzed extensively in [26]. In this section, we formally introduce a multivariate reward process associated with $J(t)$ and $[\underline{M}(t), \underline{\underline{N}}(t)]$, which would further strengthen the applicability of the UMCP as we will see.

In order to facilitate our analysis, we assume that the semi-Markov process $J(t)$ is governed by a matrix cumulative distribution function (c.d.f.) $\underline{\underline{A}}(x)=\left[A_{i j}(x)\right]$, which is assumed to be absolutely continuous with the matrix probability density function (p.d.f.) $\underline{\underline{a}}(x)=\left[a_{i j}(x)\right]=\frac{d}{d x} \underline{\underline{A}}(x)$. It should be noted that, if we define $A_{i}(x)$ and $\bar{A}_{i}(x)$ by

$$
A_{i}(x)=\sum_{j \in \mathscr{J}} A_{i j}(x) ; \quad \bar{A}_{i}(x)=1-A_{i}(x),
$$

then $A_{i}(x)$ is the c.d.f. and $\bar{A}_{i}(x)$ is the corresponding survival function of the dwell time of $J(t)$ in state $i$. The hazard rate functions associated with the semi-Markov process are then defined as $\eta_{i j}(x)=\frac{a_{i j}(x)}{\bar{A}_{i}(x)}, i, j \in \mathscr{J}$. The hazard rate function $\eta_{i j}(x)$ can be interpreted probabilistically as follows. Suppose that the semi-Markov process has been in state $i$ for the duration of $x$ since the last transition into state $i$ at time $t$. Then, for sufficiently small $\Delta>0$, the probability that the semi-Markov process makes a transition to state $j$ in the interval $(t, t+\Delta)$ can be written as $\eta_{i j}(x) \Delta+o(\Delta)$, which is independent of $t$. In other words, $\eta_{i j}(x) \Delta$ provides a linear approximation of this probability for sufficiently small $\Delta>0$, which depends only on the current dwell time $x$ in state $i$.

The Laplace transform of $\underline{\underline{a}}(x)$ is denoted by $\underline{\underline{\alpha}}(w)=\int_{0}^{\infty} e^{-w x} \underline{\underline{a}}(x) d x$.

For notational convenience, the transition epochs of the semi-Markov process are denoted by $\tau_{n}, n \geq 0$, with $\tau_{0}=0$. The age process $X(t)$ associated with the semi-Markov process is then defined as $X(t)=t-\max \left\{\tau_{n}: 0 \leq \tau_{n} \leq t\right\}$. For the cumulative arrival intensity function $L_{i}(x)$ in state $i$, one has $L_{i}(x)=\int_{0}^{x} \lambda_{i}(y) d y$. The probability of observing $k$ arrivals within the current dwell time of $x$ in state $i$ can then be obtained as

$$
g_{i}(x, k)=e^{-L_{i}(x)} \frac{L_{i}(x)^{k}}{k !}, \quad k=0,1,2, \cdots, \quad i \in \mathscr{J} .
$$


Let $\underline{Z}(t)=\left[Z_{1}(t), Z_{2}(t), \cdots, Z_{K}(t)\right]^{\top}$ be a multivariate stochastic process defined on $\mathbb{R}^{K}$ given by

$$
\underline{Z}(t)=\int_{0}^{t} \underline{\rho}(J(\tau)) d \tau+\sum_{i \in \mathscr{J}} \sum_{d=1}^{M_{i}(t)} \underline{Y}_{M: i: d}+\sum_{i \in \mathscr{J}} \sum_{j \in \mathscr{J}} \sum_{d=1}^{N_{i j}(t)} \underline{Y}_{N: i j: d},
$$

where $\rho(i)$ denotes the multivariate reward rate function while the underlying semi-Markov process $J(t)$ is in state $i \in \mathscr{J}$. $\underline{Y}_{M: i: d}$ and $\underline{Y}_{N: i j: d}$ represent the vector valued random jumps associated with the $d$-th arrival of $M_{i}(t)$ in state $i$ and the $d$-th jump of $N_{i j}(t)$ describing transitions of $J(t)$ from state $i$ to state $j$, respectively. Throughout this paper, we assume that $\underline{Y}_{M: i: d}$ are i.i.d. with respect to $d$, and so are $\underline{Y}_{N: i j: d}$. We note that $Z_{k}(t), k=1,2, \cdots, K$ are not independent. A typical sample path of the marginal reward process $Z_{k}(t), k=1,2, \cdots, K$, is depicted in Figure 1.

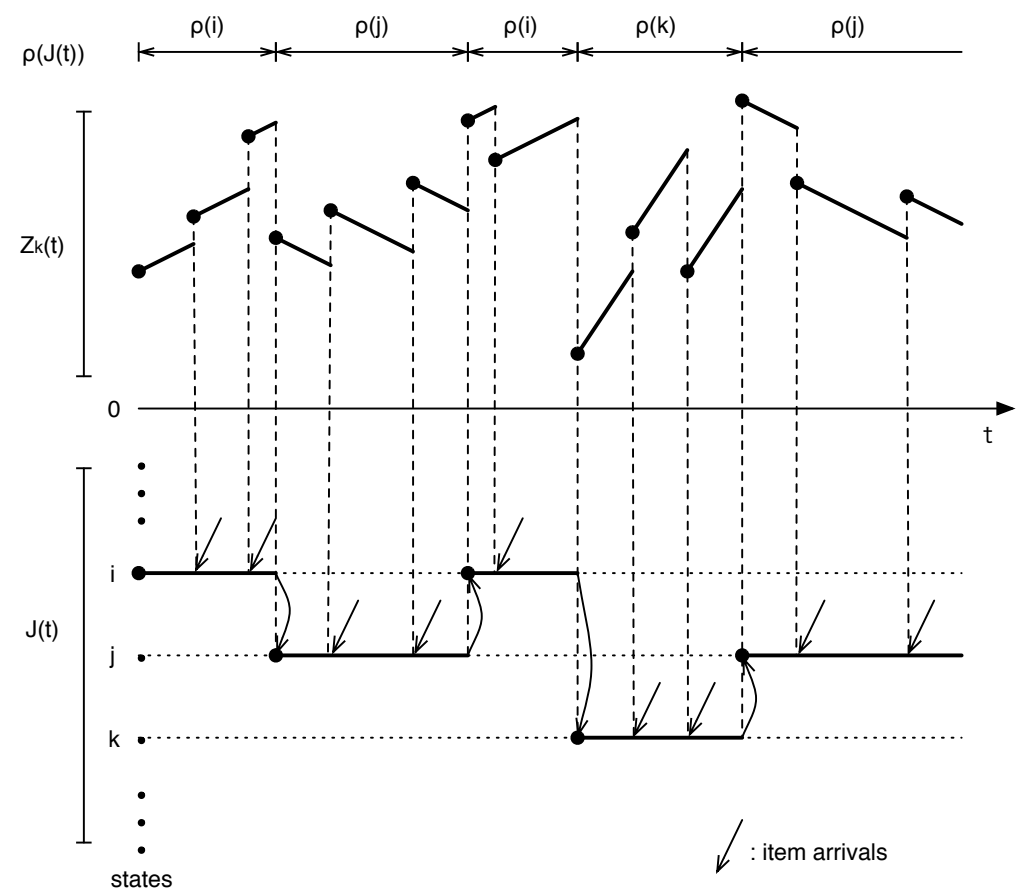

Figure 1: Typical Sample Path of the Univariate Reward Process $Z_{k}(t)$

The multivariate reward model proposed in this paper may find a variety of applications in different fields. In financial engineering, for example, the underlying semi-Markov process may describe a macro-economic condition. Corporations under consideration may be classified into several classes based on their financial strengths. It is then natural to assume that such classes have different default rates characterized by inhomogenious Poisson processes governed by $\lambda_{i}(x)$ for class $i$, where $x$ is the elapsed time since the last transition into the current macro-economic state. The reward process $Z_{i}(t)$ then describes the cumulative debt of defaulted corporations in class $i$ up to time $t$. Clearly, $Z_{i}(t)$ and $Z_{j}(t)$ are correlated and it is of interest to analyze the joint vector reward process $\underline{Z}(t)$. The underlying parameter structure in this example, however, is difficult to estimate. In this paper, we restrict ourselves to one dimensional problem in manufacturing, where the optimal preventive maintenance policy is explored for a system degrading gradually. This model is still new in that the expected profit to be maximized consists of three parts: continuous earnings during uptimes, continuous costs during 
downtimes and a random fixed cost incurred whenever a maintenance activity takes place. In addition, two performance measures are considered and compared so as to demonstrate that the optimal policy for maximizing the mean time between failures could be different from that for maximizing the system availability.

\section{Dynamic Analysis}

The purpose of this section is to derive the transform results of the multivariate reward process $\underline{Z}(t)$ through dynamic analysis. The method of supplementary variables is employed together with the Laplace transform - generating function approach. More specifically, since $\underline{Z}(t)$ is not Markov, we consider the multivariate stochastic process $[J(t), X(t), \underline{M}(t), \underline{\underline{N}}(t), \underline{Z}(t)]$ defined on the state space $S=\mathscr{J} \times \mathbb{R}_{+} \times \mathbb{Z}_{+}^{J+1} \times \mathbb{Z}_{+}^{(J+1) \times(J+1)} \times \mathbb{R}^{K}$, which is now Markov. Let $F_{i j}(x, \underline{m}, \underline{\underline{n}}, \underline{z}, t)$ be the conditional joint distribution function defined by

$$
\begin{gathered}
F_{i j}(x, \underline{m}, \underline{\underline{n}}, \underline{z}, t)=\mathrm{P}[J(t)=j, X(t) \leq x, \underline{M}(t)=\underline{m}, \underline{\underline{N}}(t)=\underline{\underline{n}}, \underline{Z}(t) \leq \underline{z} \\
J(0)=i, \underline{M}(0)=\underline{0}, \underline{\underline{N}}(0)=\underline{0}] .
\end{gathered}
$$

The following notation would be employed concerning the distributions of $\underline{Y}_{M: i: d}$ and $\underline{Y}_{N: i j: d}$ for all $d=1,2, \cdots$.

$$
\begin{array}{rlrl}
\Upsilon_{M: i}(\underline{z}) & =\mathrm{P}\left[\underline{Y}_{M: i: d} \leq \underline{z}\right] ; & \Upsilon_{N: i j}(\underline{z})=\mathrm{P}\left[\underline{Y}_{N: i j: d} \leq \underline{z}\right] \\
\mathscr{Y}_{M: i}(\underline{z})=\frac{\partial^{K}}{\partial \underline{z}} \Upsilon_{M: i}(\underline{z}) ; & \mathscr{Y}_{N: i j}(\underline{z})=\frac{\partial^{K}}{\partial \underline{z}} \Upsilon_{N: i j}(\underline{z}) \\
\theta_{M: i}(\underline{r})=\int_{\mathbb{R}^{K}} e^{-\underline{r}^{\top} \underline{z} \mathscr{Y}_{M: i}(\underline{z}) d \underline{z} ;} & \theta_{N: i j}(\underline{r})=\int_{\mathbb{R}^{K}} e^{-\underline{r}^{\top} \underline{z} \mathscr{Y}_{N: i j}(\underline{z}) d \underline{z}}
\end{array}
$$

Here, $\frac{\partial^{K}}{\partial \underline{z}}$ means that $\frac{\partial^{K}}{\partial \underline{z}}=\frac{\partial}{\partial z_{K}} \frac{\partial}{\partial z_{K-1}} \cdots \frac{\partial}{\partial z_{1}}$ and $\int_{\mathbb{R}^{K}} f(\underline{z}) d \underline{z}=\int_{\mathbb{R}^{\prime}} \cdots \int_{\mathbb{R}^{\prime}} f(\underline{z}) d z_{1} \cdots d z_{K}$.

It is natural to assume that both the age and the rewards are zero at time 0 , i.e. $X(0)=0$ and $\underline{Z}(0)=\underline{0}$ with probability 1 . A random variable and a random vector of this type have a p.d.f and a vector p.d.f. expressed as a Dirac delta function and a multivariate Dirac delta function, respectively. In this case, the differentiability of $F_{i j}(x, \underline{m}, \underline{n}, \underline{z}, t)$ with respect to $x$ and $\underline{z}$ is not present. In order to overcome this difficulty, as in Sumita and Masuda [27] and Sumita and Shanthikumar [28], we first assume that $X(0)$ and $\underline{Z}(0)$ have an absolutely continuous c.d.f. $H_{X}(x)$ and an absolutely continuous vector c.d.f. $H_{Z}(\underline{z})$ with corresponding p.d.f.s $h_{X}(x)$ and $h_{Z}(\underline{z})$, respectively. As we will see, the results for the case of $X(0)=0$ and $\underline{Z}(0)=\underline{0}$ can then be obtained by considering a sequence of absolutely continuous random variables $X_{j}$ and a sequence of absolutely continuous random vectors $\underline{Z}_{j}$ with $X_{j} \rightarrow 0$ and $\underline{Z}_{j} \rightarrow \underline{0}$ in distribution as $j \rightarrow \infty$. More formally, we define

$$
\begin{array}{ll}
H_{X}(x)=\mathrm{P}[X(0) \leq x] ; & h_{X}(x)=\frac{d}{d x} H_{X}(x), \\
H_{Z}(\underline{z})=\mathrm{P}[\underline{Z}(0) \leq \underline{z}] ; & h_{Z}(\underline{z})=\frac{\partial^{K}}{\partial \underline{z}} H_{Z}(\underline{z}) .
\end{array}
$$


The joint probability density function $f_{i j}(x, \underline{m}, \underline{n}, \underline{z}, t)$ can then be defined by

$$
f_{i j}(x, \underline{m}, \underline{\underline{n}}, \underline{z}, t)=\frac{\partial^{(K+1)}}{\partial x \partial \underline{z}} F_{i j}(x, \underline{m}, \underline{\underline{n}}, \underline{z}, t) .
$$

By examining the probabilistic flow of the multivariate process $[J(t), X(t), \underline{M}(t), \underline{\underline{N}}(t), \underline{Z}(t)]$ in its state space, one can establish the following equations:

$$
\begin{aligned}
f_{i j}(x, \underline{m}, \underline{\underline{n}}, \underline{z}, 0)= & \delta_{\{i=j\}} \delta_{\{\underline{m}=\underline{\underline{m}}\}} \boldsymbol{\delta}_{\{\underline{\underline{n}}=\underline{\underline{0}}\}} h_{X}(x) h_{Z}(\underline{z}), \\
f_{i j}(x, \underline{\underline{m}}, \underline{\underline{n}}, \underline{\underline{z}}, t)= & \delta_{\{i=j\}} \boldsymbol{\delta}_{\left\{\underline{m}=m_{i} \underline{1}_{i}\right\}} \boldsymbol{\delta}_{\{\underline{\underline{n}}=\underline{\underline{0}}\}} h_{X}(x-t) \cdot \mathbf{A}(t) \cdot \frac{\bar{A}_{i}(x)}{\bar{A}_{i}(x-t)} g_{i}\left(t, m_{i}\right) \\
& +\left(1-\boldsymbol{\delta}_{\{\underline{\underline{n}}=\underline{\underline{0}}\}}\right) \sum_{k=0}^{m_{j}}\left\{\mathbf{B}_{k}(x, t) \cdot \bar{A}_{j}(x) g_{j}(x, k)\right\}, \\
f_{i j}(0+, \underline{m}, \underline{\underline{n}}, \underline{z}, t)= & \left(1-\delta_{\{\underline{\underline{n}}=\underline{\underline{0}}\}}\right) \sum_{\ell \in \mathscr{J}} \mathbf{C}_{\ell}(x, t),
\end{aligned}
$$

where

$$
\begin{aligned}
& \mathbf{A}(t)=\underbrace{\int_{\mathbb{R}^{K}} \cdots \int_{\mathbb{R}^{K}}}_{m_{i}} h_{Z}\left(\underline{z}-\underline{\rho}(i) t-\sum_{d=1}^{m_{i}} \underline{z}_{M: i: d}\right) \prod_{d=1}^{m_{i}} \mathscr{Y}_{M: i}\left(\underline{z}_{M: i: d}\right) \underbrace{d \underline{z_{M: i: 1} \cdots d \underline{z}_{M: i: m_{i}}}}_{m_{i}}, \\
& \mathbf{B}_{k}(x, t)=\underbrace{\int_{\mathbb{R}^{K}} \cdots \int_{\mathbb{R}^{K}}}_{k} f_{i j}\left(0+, \underline{m}-k \underline{1}_{j}, \underline{\underline{n}}, \underline{z}-\underline{\rho}(j) x-\sum_{d=m_{j}-k+1}^{m_{j}} \underline{z}_{M: j: d}, t-x\right) \\
& \times \prod_{d=m_{j}-k+1}^{m_{j}} \mathscr{Y}_{M: j}\left(\underline{z}_{M: j: d}\right) \underbrace{d \underline{z}_{M: j: m_{j}-k+1} \cdots d \underline{z}_{M: j: m_{j}}}_{k}, \\
& \mathbf{C}_{\ell}(x, t)=\int_{\mathbb{R}^{K}} \int_{0}^{\infty} f_{i \ell}\left(x, \underline{m}, \underline{\underline{n}}-\underline{\underline{1}}_{\ell j}, \underline{z}-\underline{z}_{N: \ell j: n_{\ell j}}, t\right) \mathscr{Y}_{N: \ell j}\left(\underline{z}_{N: \ell j: n_{\ell j}}\right) \eta_{\ell j}(x) d x d \underline{z}_{N: \ell j: n_{\ell j}} .
\end{aligned}
$$

Eq. (3.6) describes the initial condition at time $t=0$ with $\underline{M}(0)=\underline{0}, \underline{N}(0)=\underline{0}$ and $i=j$. The first term on the right hand side of Eq. (3.7) represents the following scenario. During the time interval $(0, t]$, the underlying semi-Markov process $J(t)$ has not left its initial state $i$, and there has been $m_{i}$ arrivals of $M_{i}(\cdot)$. Furthermore, the current age in state $i$ at time $t$ is $x$ with the cumulative reward in the interval $(0, t]$ described by $\mathbf{A}(t)$. For the second term on the right hand side of Eq. (3.7), we focus on the probabilistic flow of the multivariate process $[J(t), X(t), \underline{M}(t), \underline{\underline{N}}(t), \underline{Z}(t)]$. For this process to be in state $[\underline{m}, \underline{n}, \underline{z}, x, t]$ at time $t$ having at least one transition of the semi-Markov process occurred in $(0, t]$ with $J(t)=j$, the semi-Markov process must have entered state $j \in \mathscr{J}$ at time $t-x$ with $\left[0+, \underline{m}-k \underline{1}_{j}, \underline{\underline{n}}, \underline{z}-\underline{\rho}(j) x-\sum_{d=m_{j}-k+1}^{m_{j}} \underline{z}_{M: j: d}, t-x\right]$, no transition of the semi-Markov process has occurred in $(t-x, t]$ with this probability being $\bar{A}_{j}(x)$, there have been $k$ arrivals of $M_{j}(\cdot)$ in $(t-x, t]$ with probability $g_{j}(x, k)$ for $k=0,1, \cdots, m_{j}$, and the reward of $\underline{\rho}(j) x+\sum_{d=m_{j}-k+1}^{m_{j}} \underline{z}_{M: j: d}$ has been accumulated with probability $\mathbf{B}_{k}(x, t)$, for $k=0,1, \cdots, m_{j}$.

Eq. (3.8) describes the boundary condition for $X(t)$ at $x=0+$. Namely, the multivariate process $[J(t), X(t), \underline{M}(t), \underline{\underline{N}}(t), \underline{Z}(t)]$ can enter the state $[0+, \underline{m}, \underline{n}, \underline{z}, t]$ at time $t$ with $J(t)=j$ from $[x, \underline{m}, \underline{\underline{n}}-$ $\left.\underline{\underline{1}}_{\ell j}, \underline{z}_{-}-\underline{z}_{N: \ell j: n_{\ell j}}, t\right]$ with the state of the semi-Markov process being $\ell \in \mathscr{J}$, only when the current 
age expires with the hazard rate $\eta_{\ell j}(x)$ and the reward associated with this jump of the semi-Markov process from $\ell$ to $j$ is $\underline{z}_{N: \ell j: n_{\ell j}}$ which happens with the p.d.f. $\mathscr{Y}_{N: \ell j}\left(\underline{z}_{N: \ell j: n_{\ell j}}\right)$.

In order to solve the functional equations in (3.6) through (3.11), we introduce the following Laplace transform generating functions.

$$
\begin{aligned}
& \underline{\underline{\beta}}(w, m)=\left[\beta_{i j}(w, m)\right] ; \quad \beta_{i j}(w, m)=\int_{0}^{\infty} e^{-w x} a_{i j}(x) g_{i}(x, m) d x, \\
& \underline{\underline{\beta}}(\underline{w}, \underline{u})=\left[\widehat{\beta}_{i j}\left(w_{i}, u_{i}\right)\right] ; \quad \widehat{\beta}_{i j}\left(w_{i}, u_{i}\right)=\sum_{m_{i}=0}^{\infty} \beta_{i j}\left(w_{i}, m_{i}\right) u_{i}^{m_{i}}, \\
& \underline{\underline{\beta}}_{D}^{*}(w, m)=\left[\delta_{\{i=j\}} \beta_{i}^{*}(w, m)\right] ; \quad \beta_{i}^{*}(w, m)=\int_{0}^{\infty} e^{-w x} \bar{A}_{i}(x) g_{i}(x, m) d x, \\
& \underline{\underline{\beta}}_{D}^{*}(\underline{w}, \underline{u})=\left[\delta_{\{i=j\}} \widehat{\beta}_{i}^{*}\left(w_{i}, u_{i}\right)\right] ; \quad \widehat{\beta}_{i}^{*}\left(w_{i}, u_{i}\right)=\sum_{m_{i}=0}^{\infty} \beta_{i}^{*}\left(w_{i}, m_{i}\right) u_{i}^{m_{i}}, \\
& \underline{\underline{\rho}}^{*}(s, m)=\left[\rho_{i j}^{*}(s, m)\right] ; \quad \rho_{i j}^{*}(s, m)=\int_{0}^{\infty} e^{-s t} g_{i}(t, m) \int_{0}^{\infty} h_{X}(x-t) \frac{a_{i j}(x)}{\bar{A}_{i}(x-t)} d x d t, \\
& \underline{\underline{\rho}}^{*}(\underline{s}, \underline{u})=\left[\widehat{\rho}_{i j}^{*}\left(s_{i}, u_{i}\right)\right] ; \quad \widehat{\rho}_{i j}^{*}\left(s_{i}, u_{i}\right)=\sum_{m_{i}=0}^{\infty} \rho_{i j}^{*}\left(s_{i}, m_{i}\right) u_{i}^{m_{i}}, \\
& \mathscr{K}_{Z}(\underline{r})=\int_{\mathbb{R}^{K}} e^{-\underline{r}^{\top} \underline{z}} h_{Z}(\underline{z}) d \underline{z}, \\
& \underline{\theta}_{M}(\underline{r})=\left[\theta_{M: i}(\underline{r})\right] ; \quad \theta_{M: i}(\underline{r})=\int_{\mathbb{R}^{K}} e^{-\underline{r}^{\top} \underline{z}_{M}} \mathscr{Y}_{M: i}\left(\underline{z}_{M}\right) d \underline{z}_{M}, \\
& \underline{\theta}_{N}(\underline{r})=\left[\theta_{N: i j}(\underline{r})\right] ; \quad \theta_{N: i j}(\underline{r})=\int_{\mathbb{R}^{K}} e^{-\underline{r}^{\top} \underline{z}_{N}} \mathscr{Y}_{N: i j}\left(\underline{z}_{N}\right) d \underline{z}_{N} \\
& \underline{\underline{\xi}}(\underline{m}, \underline{\underline{n}}, \underline{r}, s)=\left[\xi_{i j}(\underline{m}, \underline{\underline{n}}, \underline{r}, s)\right] ; \quad \xi_{i j}(\underline{m}, \underline{\underline{n}}, \underline{r}, s)=\int_{0}^{\infty} e^{-s t} \int_{\mathbb{R}^{K}} e^{-\underline{r}^{\top} \underline{z}} f_{i j}(0+, \underline{m}, \underline{\underline{n}}, \underline{z}, t) d \underline{z} d t, \\
& \underline{\underline{\xi}}(\underline{u}, \underline{\underline{v}}, \underline{r}, s)=\left[\widehat{\xi}_{i j}(\underline{u}, \underline{\underline{v}}, \underline{r}, s)\right] ; \quad \widehat{\xi}_{i j}(\underline{u}, \underline{\underline{v}}, \underline{r}, s)=\sum_{\underline{\underline{n}} \mathbb{Z}_{+}^{(J+1)^{2}} \backslash\{\underline{\underline{0}}\} \underline{\underline{m} \in \mathbb{Z}_{+}^{J+1}}} \xi_{i j}(\underline{m}, \underline{\underline{n}}, \underline{r}, s) \underline{\underline{u}} \underline{\underline{m}} \underline{\underline{v}} \underline{\underline{\underline{n}}}, \\
& \underline{\underline{\varphi}}(w, \underline{m}, \underline{\underline{n}}, \underline{r}, s)=\left[\varphi_{i j}(w, \underline{m}, \underline{\underline{n}}, \underline{r}, s)\right] \\
& \varphi_{i j}(w, \underline{m}, \underline{\underline{n}}, \underline{r}, s)=\int_{0}^{\infty} e^{-w x} \int_{0}^{\infty} e^{-s t} \int_{\mathbb{R}^{K}} e^{-\underline{r}^{\top}} \underline{z} f_{i j}(x, \underline{m}, \underline{\underline{n}}, \underline{z}, t) d \underline{z} d t d x, \\
& \underline{\underline{\hat{\varphi}}}(w, \underline{u}, \underline{\underline{v}}, \underline{r}, s)=\left[\widehat{\varphi}_{i j}(w, \underline{u}, \underline{\underline{v}}, \underline{r}, s)\right] ; \quad \widehat{\varphi}_{i j}(w, \underline{u}, \underline{\underline{v}}, \underline{r}, s)=\sum_{\underline{\underline{n}} \in \mathbb{Z}_{+}^{(J+1)^{2}}} \sum_{\underline{m} \in \mathbb{Z}_{+}^{J+1}} \varphi_{i j}(w, \underline{m}, \underline{\underline{n}}, \underline{r}, s) \underline{u}^{\underline{\underline{m}}} \underline{\underline{v}}^{\underline{\underline{n}}},
\end{aligned}
$$

where $\underline{u}^{\underline{m}}=\prod_{i \in \mathscr{J}} u_{i}^{m_{i}}$ and $\underline{\underline{\underline{n}}} \underline{\underline{\underline{n}}}=\prod_{\{i, j\} \in \mathscr{J}^{2} \backslash\{(0,0)\}} v_{i j}^{n_{i j}}$. It should be noted that these functionals are introduced in order to facilitate our analysis, given the exogenous inputs $a_{i j}(x), \bar{A}_{i}(x)$ and $g_{i}(x, m)$ in Eq.'s (3.12) and (3.13). 
We are now in a position to establish the main theorem of this section. For notational convenience, the operator $\otimes$ is defined as the component-wise multiplication between two vectors or two matrices of the same size, i.e. for $\underline{a}=\left[a_{1}, \cdots, a_{i}, \cdots a_{n}\right]$ and $\underline{b}=\left[b_{1}, \cdots, b_{i}, \cdots b_{n}\right]$, we define $\underline{a} \otimes \underline{b}=\left[a_{1} \times\right.$ $\left.b_{1}, \cdots, a_{i} \times b_{i}, \cdots, a_{n} \times b_{n}\right]$. Similarly, for two matrices $\underline{\underline{A}}=\left[a_{i j}\right]$ and $\underline{\underline{B}}=\left[b_{i j}\right]$, we write $\underline{\underline{A}} \otimes \underline{\underline{B}}=$ $\left[a_{i j} \times b_{i j}\right]$. It is worth noting that, for $\underline{\gamma}=\left[\gamma_{0}, \cdots, \gamma_{J}\right]^{\top}$ with $\gamma_{i}=\underline{r}^{\top} \underline{\rho}(i)$, the inverse matrix

$$
\left\{\underline{\underline{I}}-\underline{\underline{v}} \otimes \underline{\underline{\theta}}_{N}(\underline{r}) \otimes \underline{\underline{\beta}}\left(\underline{\gamma}+s \underline{1}, \underline{u} \otimes \underline{\theta}_{M}(\underline{r})\right)\right\}^{-1}
$$

always exists for $\underline{r}$ on the unit sphere in the $K$ dimensional complex space, $\underline{\underline{v}}=\left[v_{i j}\right]$ with $\left|v_{i j}\right| \leq 1$, $\underline{u}=\left[u_{i}\right]$ with $\left|u_{i}\right| \leq 1$ and $\operatorname{Re}(s)>0$. This can be seen by observing that, with $\underline{\underline{\omega}}=\underline{\underline{v}} \otimes \underline{\underline{\theta}}_{N}(\underline{r}) \otimes \underline{\underline{\beta}}(\underline{\gamma}+$ $\left.s \underline{1}, \underline{u} \otimes \underline{\theta}_{M}(\underline{r})\right)$,

$$
\omega_{i j}=v_{i j} \times \theta_{N: i j}(\underline{r}) \times \widehat{\beta}_{i j}\left(\gamma_{i}+s, u_{i} \times \theta_{M: i}(\underline{r})\right)
$$

where, from (3.12),

$$
\widehat{\beta}_{i j}\left(\gamma_{i}+s, u_{i} \times \theta_{M: i}(\underline{r})\right)=\sum_{m_{i}=0}^{\infty}\left(\int_{0}^{\infty} e^{-\left(\gamma_{i}+s\right) x} a_{i j}(x) g_{i}\left(x, m_{i}\right) d x\right) \times\left(u_{i} \times \theta_{M: i}(\underline{r})\right)^{m_{i}}
$$

For $\underline{r}, \underline{\underline{v}}, \underline{u}$ and $s$ in the range specified above, one sees that $\left|\omega_{i j}\right|<\int_{0}^{\infty} a_{i j}(x) d x$. Consequently, $\underline{\underline{\omega}}$ is strictly dominated by the stochastic matrix $\left[\int_{0}^{\infty} a_{i j}(x) d x\right]$, and therefore the spectral radius of $\underline{\underline{\underline{\omega}}}$ is strictly less than 1 . This in turn implies that $[\underline{\underline{I}}-\underline{\underline{\omega}}]^{-1}$ exists. We now state the main theorem.

Theorem 3.1. Let $X(0)=0$ and $\underline{Z}(0)=\underline{0}$ with probability one. Then,

$$
\begin{aligned}
& \underline{\underline{\hat{\xi}}}(\underline{u}, \underline{\underline{v}}, \underline{r}, s)=\left\{\underline{\underline{v}} \otimes \underline{\underline{\theta}}_{N}(\underline{r}) \otimes \underline{\underline{\beta}}\left(\underline{\gamma}+s \underline{\underline{1}}, \underline{u} \otimes \underline{\theta}_{M}(\underline{r})\right)\right\} \times\left\{\underline{\underline{I}}-\underline{\underline{v}} \otimes \underline{\underline{\theta}}_{N}(\underline{r}) \otimes \underline{\underline{\beta}}\left(\underline{\gamma}+s \underline{1}, \underline{u} \otimes \underline{\theta}_{M}(\underline{r})\right)\right\}^{-1} ; \\
& \underline{\underline{\hat{\varphi}}}(w, \underline{u}, \underline{\underline{v}}, \underline{r}, s)=\left\{\underline{\underline{I}}-\underline{\underline{v}} \otimes \underline{\underline{\theta}}_{N}(\underline{r}) \otimes \underline{\underline{\beta}}\left(\underline{\gamma}+s \underline{1}, \underline{u} \otimes \underline{\theta}_{M}(\underline{r})\right)\right\}^{-1} \times \underline{\underline{\beta}}_{D}^{*}\left(\underline{\gamma}+(w+s) \underline{1}, \underline{u} \otimes \underline{\theta}_{M}(\underline{r})\right),
\end{aligned}
$$

where $\underline{\gamma}=\left[\gamma_{0}, \cdots, \gamma_{J}\right]^{\top}$ with $\gamma_{i}=\underline{r}^{\top} \underline{\rho}(i)$. 
Proof We first assume that $X(0)$ and $\underline{Z}(0)$ have p.d.f.s $h_{X}(x)$ and $h_{Z}(\underline{z})$ respectively. Substituting Eq.'s (3.6) and (3.7) into (3.8), one sees that

$$
\begin{aligned}
& f_{i j}(0+, \underline{m}, \underline{\underline{n}}, \underline{z}, t)
\end{aligned}
$$

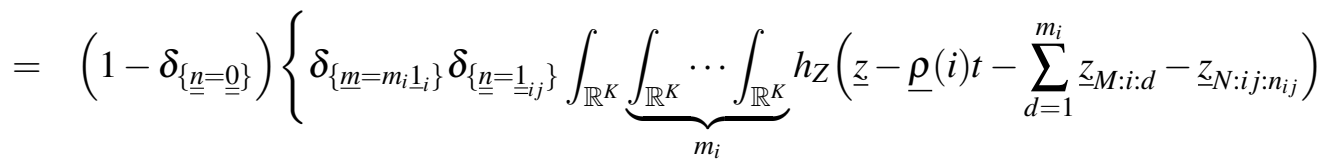

$$
\begin{aligned}
& \times \quad \prod_{d=1}^{m_{i}} \mathscr{Y}_{M: i}\left(\underline{z}_{M: i: d}\right) \mathscr{Y}_{N: i j}\left(\underline{z}_{N: i j: n_{i j}}\right) \underbrace{d \underline{z}_{M: i: 1} \cdots d \underline{z}_{M: i: m_{i}}}_{m_{i}} d \underline{z}_{N: i j: n_{i j}} \\
& \left.\times \quad g_{i}\left(t, m_{i}\right) \int_{0}^{\infty} h_{X}(x-t) \frac{a_{i j}(x)}{\bar{A}_{i}(x-t)} d x\right\} \\
& +\left(1-\delta_{\{\underline{\underline{n}}=\underline{\underline{0}}\}}\right) \sum_{\ell \in \mathscr{J}}\left(1-\delta_{\left\{\underline{\underline{n}}=\underline{\underline{1}} \underline{i}_{j}\right\}}\right) \sum_{k=0}^{m_{\ell}}\{\int_{0}^{\infty} \int_{\mathbb{R}^{K}} \underbrace{\int_{\mathbb{R}^{K}} \cdots \int_{\mathbb{R}^{K}}}_{k} \\
& f_{i \ell}\left(0+, \underline{m}-r \underline{1}_{\ell}, \underline{\underline{n}}-\underline{\underline{1}}_{\ell j}, \underline{z}-\underline{\rho}(\ell) x-\sum_{d=m_{j}-k+1}^{m_{j}} \underline{z}_{M: j: d}-\underline{z}_{N: \ell j: n_{\ell j}}, t-x\right) \\
& \times \prod_{d=m_{j}-k+1}^{m_{j}} \mathscr{Y}_{M: j}\left(\underline{z}_{M: j: d}\right) \mathscr{Y}_{N: \ell j}\left(\underline{z}_{N: \ell j: n_{\ell j}}\right) \underbrace{d \underline{z}_{M: j: m_{j}-k+1} \cdots d \underline{z}_{M:: m_{j}}}_{k} d \underline{z}_{N: \ell j: n_{\ell j}} \\
& \left.\times \quad a_{\ell j}(x) g_{\ell}(x, k) d x\right\} .
\end{aligned}
$$

By taking the Laplace transform of both sides of the above equation with respect to $\underline{z}$ and $t$, it follows that

$$
\begin{aligned}
& \xi_{i j}(\underline{m}, \underline{\underline{n}}, \underline{r}, s) \\
& =\left(1-\delta_{\{\underline{\underline{n}}=\underline{0}\}}\right)\left\{\delta _ { \{ \underline { m } = m _ { i } \underline { 1 } _ { i } \} } \delta _ { \{ \underline { \underline { n } } = \underline { \underline { 1 } } _ { i j } \} } \mathscr { K } _ { Z } ( \underline { r } ) \theta _ { M : i } \left(\underline{r}^{m_{i}} \theta_{N: i j}(\underline{r}) \rho_{i j}^{*}\left(\underline{r}^{\top} \underline{\rho}(i)+s, m_{i}\right)\right.\right. \\
& +\sum_{\ell \in \mathscr{J}}\left(1-\delta_{\left\{\underline{\underline{n}}-\underline{\underline{1}}_{\ell j}\right\}}\right) \sum_{k=0}^{m_{\ell}}\left[\xi_{i \ell}\left(\underline{m}-r \underline{\underline{1}}_{\ell}, \underline{\underline{n}}-\underline{\underline{1}}_{\ell j}, \underline{r}, s\right) \theta_{M: \ell}(\underline{r})^{k} \theta_{N: \ell j}(\underline{\underline{v}})\right. \\
& \left.\left.\quad \times \beta_{\ell j}\left(\underline{r}^{\top} \underline{\rho}(\ell)+s, k\right)\right]\right\} .
\end{aligned}
$$

Multiplying $\underline{u}^{\underline{m}}$ and $\underline{\underline{\underline{\nu}}} \underline{\underline{n}}$ to both sides and summing over $\underline{m} \geq \underline{0}$ and $\underline{\underline{n}} \geq \underline{\underline{0}}$, this equation then leads to

$$
\begin{aligned}
\underline{\underline{\hat{\xi}}}(\underline{u}, \underline{\underline{v}}, \underline{r}, s)= & \mathscr{K}_{Z}(\underline{r}) \cdot \underline{\underline{v}} \otimes \underline{\underline{\theta}}_{N}(\underline{r}) \otimes \underline{\underline{\rho}}^{*}\left(\underline{\gamma}+s \underline{1}, \underline{u} \otimes \underline{\theta}_{M}(\underline{r})\right) \\
& +\underline{\underline{\xi}}(\underline{u}, \underline{\underline{v}}, \underline{r}, s)\left\{\underline{\underline{v}} \otimes \underline{\underline{\theta}}_{N}(\underline{r}) \otimes \underline{\hat{\beta}}\left(\underline{\gamma}+s \underline{1}, \underline{u} \otimes \underline{\theta}_{M}(\underline{r})\right)\right\},
\end{aligned}
$$


which can be solved for $\underline{\underline{\xi}}(\underline{u}, \underline{\underline{v}}, \underline{r}, s)$, yielding

$$
\begin{aligned}
\underline{\underline{\xi}}(\underline{u}, \underline{\underline{v}}, \underline{r}, s)= & \mathscr{K}_{Z}(\underline{r})\left\{\underline{\underline{v}} \otimes \underline{\underline{\theta}}_{N}(\underline{r}) \otimes \underline{\widehat{\rho}}^{*}\left(\underline{\gamma}+s \underline{1}, \underline{u} \otimes \underline{\theta}_{M}(\underline{r})\right)\right\} \\
& \times\left\{\underline{\underline{I}}-\underline{\underline{v}} \otimes \underline{\underline{\theta}}_{N}(\underline{r}) \otimes \underline{\underline{\beta}}\left(\underline{\gamma}+s \underline{1}, \underline{u} \otimes \underline{\theta}_{M}(\underline{r})\right)\right\} .
\end{aligned}
$$

Let

$$
\varepsilon_{i}(w, s, m)=\int_{0}^{\infty} e^{-w x} \int_{0}^{\infty} e^{-s t} h_{X}(x-t) \frac{\bar{A}_{i}(x)}{\bar{A}_{i}(x-t)} g_{i}(t, m) d t d x
$$

and define the diagonal matrix

$$
\underline{\widehat{\varepsilon}}_{D}(w, \underline{s}, \underline{u})=\left[\delta_{\{i=j\}} \widehat{\varepsilon}_{i}\left(w, s_{i}, u_{i}\right)\right] ; \quad \widehat{\varepsilon}_{i}\left(w, s_{i}, u_{i}\right)=\sum_{m_{i}=0}^{\infty} \varepsilon_{i}\left(w, s_{i}, m_{i}\right) u_{i}^{m_{i}} .
$$

Through similar Laplace transform operations applied to (3.7), one finds that

$$
\begin{aligned}
& \varphi_{i j}(w, \underline{m}, \underline{\underline{n}}, \underline{r}, s) \\
& =\delta_{\{i=j\}} \delta_{\left\{\underline{m}=m_{i} \underline{1}_{i}\right\}} \delta_{\{\underline{\underline{n}}=\underline{\underline{0}}\}} \mathscr{K}_{Z}(\underline{r}) \theta_{M: i}(\underline{r})^{m_{i}} \varepsilon_{i}\left(w, \underline{r}^{\top} \underline{\rho}(i)+s, m_{i}\right) \\
& \quad+\left(1-\delta_{\{\underline{\underline{n}}=\underline{\underline{n}}\})}\right) \sum_{k=0}^{m_{j}} \theta_{M: j}(\underline{r})^{k} \xi_{i j}\left(\underline{m}-r \underline{1}_{j}, \underline{\underline{n}}, \underline{r}, s\right) \beta_{j}^{*}\left(\underline{r}^{\top} \underline{\rho}(j)+w+s, k\right) .
\end{aligned}
$$

Taking the generating functions with respect to $\underline{m}$ and $\underline{\underline{n}}$ as before, the following matrix equation can be obtained.

$$
\begin{aligned}
\underline{\underline{\hat{\varphi}}}(w, \underline{u}, \underline{v}, \underline{r}, s)= & \mathscr{K}_{Z}(\underline{r}) \underline{\widehat{\varepsilon}}_{D}\left(w, \underline{\gamma}+s \underline{1}, \underline{u} \otimes \underline{\theta}_{M}((v))\right) \\
& +\underline{\underline{\xi}}(\underline{u}, \underline{\underline{v}}, \underline{r}, s) \underline{\widehat{\beta}}_{D}^{*}\left(\underline{\gamma}+(w+s) \underline{1}, \underline{u} \otimes \underline{\theta}_{M}(\underline{r})\right)
\end{aligned}
$$

As in Sumita and Masuda [27] and Sumita and Shanthikumar [28], we now consider a sequence of random variables $X(0)$ converging to 0 in distribution, as well as a sequence of random vectors $\underline{Z}(0)$ with convergence to $\underline{0}$ in distribution. In other words, we let $H_{X}(x) \rightarrow U(x)$ and $H_{Z}(\underline{z}) \rightarrow U(\underline{z})$, where $U(x)$ and $U(\underline{z})$ denotes the univariate and the multivariate Heaviside step functions respectively, i.e. $U(x)=1$ for $x \geq 0$ and $U(x)=0$ otherwise. Similarly, $U(\underline{z})=1$ for $\underline{z} \geq \underline{0}$ and $U(\underline{z})=0$ otherwise. It then follows that $\mathscr{K}_{Z}(\underline{r}) \rightarrow 1, \rho_{i j}^{*}(s, m) \rightarrow \beta_{i j}(s, m)$ and $\varepsilon_{i}(w, s, m) \rightarrow \beta_{i}^{*}(w+s, m)$ from their definitions. Substituting these into Eq.'s (3.14) and (3.17), one sees that

$$
\begin{aligned}
\underline{\underline{\xi}}(\underline{u}, \underline{\underline{v}}, \underline{r}, s)= & \left\{\underline{\underline{v}} \otimes \underline{\underline{\theta}}_{N}(\underline{r}) \otimes \underline{\underline{\beta}}\left(\underline{\gamma}+s \underline{1}, \underline{u} \otimes \underline{\theta}_{M}(\underline{r})\right)\right\} \\
& \times\left\{\underline{\underline{I}}-\underline{\underline{v}} \otimes \underline{\underline{\theta}}_{N}(\underline{r}) \otimes \underline{\underline{\beta}}\left(\underline{\gamma}+s \underline{1}, \underline{u} \otimes \underline{\theta}_{M}(\underline{r})\right)\right\}^{-1} ; \\
\underline{\underline{\hat{\varphi}}}(w, \underline{u}, \underline{\underline{v}}, \underline{r}, s)= & \left\{\underline{\underline{I}}-\underline{\underline{v}} \otimes \underline{\underline{\theta}}_{N}(\underline{r}) \otimes \underline{\hat{\beta}}\left(\underline{\gamma}+s \underline{1}, \underline{u} \otimes \underline{\theta}_{M}(\underline{r})\right)\right\}^{-1} \\
& \times \underline{\underline{\beta}}_{D}^{*}\left(\underline{\gamma}+(w+s) \underline{1}, \underline{u} \otimes \underline{\theta}_{M}(\underline{r})\right),
\end{aligned}
$$


completing the proof.

Theorem 3.1 is very informative because it contains all the knowledge about the joint distribution of the multivariate process $[J(t), X(t), \underline{M}(t), \underline{\underline{N}}(t), \underline{Z}(t)]$. One can use this theorem, for example, to derive the asymptotic behaviors as $t \rightarrow \infty$ of the expectations of various processes expressed by $\underline{Z}(t)$, e.g. $\mathrm{E}\left[\prod_{k=1}^{K} Z_{k}(t)\right]$, as we will see.

Remark 3.2. We note that, when $Y_{M: i: d}=0$ and $Y_{N: i j: d}=0$, one has

$$
\underline{Z}(t)=\int_{0}^{t} \underline{\rho}(J(\tau)) d \tau
$$

so that $\underline{\underline{\hat{\varphi}}}(w, \underline{1}, \underline{\underline{1}}, \underline{r}, s)$ of Theorem 3.1 is reduced to Theorem 2.1 of Masuda and Sumita [17]. With random increments of the reward at the times of jumps of $N_{i j}(t)$, by setting $Y_{M: i: d}=0$, it can be seen that

$$
\underline{Z}(t)=\int_{0}^{t} \underline{\rho}(J(\tau)) d \tau+\sum_{i \in \mathscr{J}} \sum_{j \in \mathscr{J}} \sum_{d=1}^{N_{i j}(t)} \underline{Y}_{N: i j: d},
$$

and $\underline{\underline{\varphi}}(0+, \underline{1}, \underline{\underline{v}}, \underline{r}, s)$ is also reduced to Eq. (3.2) of Masuda [15], demonstrating that Theorem 3.1 generalizes the results of Masuda and Sumita [17] and Masuda [15].

\section{Asymptotic Behavior of $\mathbf{E}\left[\prod_{k=1}^{K} Z_{k}(t)\right]$ as $t \rightarrow \infty$}

In this section, we discuss the asymptotic behavior of $\mathrm{E}\left[\prod_{k=1}^{K} Z_{k}(t)\right]$ as $t \rightarrow \infty$. For notational convenience, let $\underline{\underline{A}}_{d}=\int_{0}^{\infty} x^{d} \underline{\underline{a}}(x) d x, d=0,1,2, \cdots$, with $\left\|\underline{\underline{A}}_{d}\right\|<\infty$ for $0 \leq d \leq K+2$, where $K$ is the dimension of vector $\underline{Z}(t)$. We assume that the Markov chain in discrete time governed by the stochastic matrix $\underline{\underline{A}}_{0}$ is irreducible, where $\underline{e}^{\top}$ is the eigenvector of $\underline{\underline{A}}_{0}$ with eigenvalue 1 so that $\underline{e}^{\top} \underline{\underline{A}}_{0}=\underline{e}^{\top}$ and $\underline{e}^{\top} \underline{1}=1$. We also define $\underline{\underline{A}}_{D: d}^{*}=\int_{0}^{\infty} x^{d} \underline{\underline{A}}_{D}(x) d x, d=0,1,2, \cdots$ with $\left\|\underline{A}_{D: d}^{*}\right\|<\infty$ for $0 \leq d \leq K+2$.

It has been seen in Theorem 3.1 that

$$
\underline{\underline{\hat{\varphi}}}(w, \underline{u}, \underline{\underline{v}}, \underline{r}, s)=\left\{\underline{\underline{I}}-\underline{\underline{v}} \otimes \underline{\underline{\theta}}_{N}(\underline{r}) \otimes \underline{\underline{\beta}}\left(\underline{\gamma}+s \underline{1}, \underline{u} \otimes \underline{\theta}_{M}(\underline{r})\right)\right\}^{-1} \times \underline{\underline{\beta}}_{D}^{*}\left(\underline{\gamma}+(w+s) \underline{1}, \underline{u} \otimes \underline{\theta}_{M}(\underline{r})\right)
$$

where $\underline{\gamma}=\left[\gamma_{0}, \cdots, \gamma_{J}\right]^{\top}$ with $\gamma_{i}=\underline{r}^{\top} \underline{\rho}(i)$. Let $\underline{p}(0)$ be an initial probability vector of $J(0)$. It then follows that

$$
\begin{aligned}
\mathscr{L}\left\{\mathrm{E}\left[e^{-\underline{r}^{\top} \underline{Z}(t)}\right]\right\} & =\underline{p}^{\top}(0) \times \underline{\underline{\hat{\varphi}}}(0+, \underline{1}, \underline{\underline{1}}, \underline{r}, s) \times \underline{1} \\
& =\underline{p}^{\top}(0) \times\left\{\underline{\underline{I}}-\underline{\underline{\theta}}_{N}(\underline{r}) \otimes \underline{\hat{\beta}}\left(\underline{\gamma}+s \underline{1}^{\prime} \underline{\theta}_{M}(\underline{r})\right)\right\}^{-1} \times \underline{\underline{\beta}}_{D}^{*}\left(\underline{\gamma}+s \underline{1}, \underline{\theta}_{M}(\underline{r})\right) \times \underline{1} .
\end{aligned}
$$

From Eq.'s (3.4), (3.12) and (3.13), one has

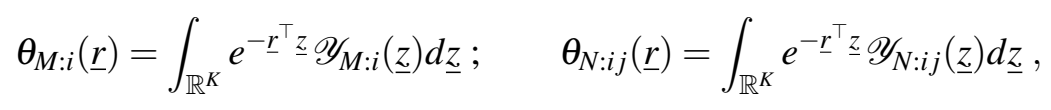




$$
\widehat{\beta}_{i j}\left(\underline{r}^{\top} \underline{\rho}(i)+s, \theta_{M: i}(\underline{r})\right)=\int_{0}^{\infty} e^{-\left\{\underline{r}^{\top} \underline{\rho}(i)+s\right\} t} e^{-L_{i}(t)\left\{1-\theta_{M: i}(\underline{r})\right\}} a_{i j}(t) d t
$$

and

$$
\widehat{\beta}_{i}^{*}\left(\underline{r}^{\top} \underline{\rho}(i)+s, \theta_{M: i}(\underline{r})\right)=\int_{0}^{\infty} e^{-\left\{\underline{r}^{\top} \underline{\rho}(i)+s\right\} t} e^{-L_{i}(t)\left\{1-\theta_{M: i}(\underline{r})\right\}} \bar{A}_{i}(t) d t .
$$

The first order partial derivatives of $\theta_{M: i}(\underline{r})$ and $\theta_{N: i j}(\underline{r})$ at $\underline{r}=\underline{0}$ are then given by

$$
\begin{gathered}
\left.\Theta_{M: i} \stackrel{\text { def }}{=} \frac{\partial^{K}}{\partial \underline{r}} \theta_{M: i}(\underline{r})\right|_{\underline{r}=\underline{0}}=(-1)^{K} \int_{\mathbb{R}^{K}} \prod_{k=1}^{K} z_{k} \mathscr{Y}_{M: i}(\underline{z}) d \underline{z}, \\
\left.\Theta_{N: i j} \stackrel{\text { def }}{=} \frac{\partial^{K}}{\partial \underline{r}} \theta_{N: i j}(\underline{r})\right|_{\underline{r}=\underline{0}}=(-1)^{K} \int_{\mathbb{R}^{K}} \prod_{k=1}^{K} z_{k} \mathscr{Y}_{N: i j}(\underline{z}) d \underline{z} .
\end{gathered}
$$

Furthermore, the first order partial derivatives of $\widehat{\beta}_{i j}\left(\underline{r}^{\top} \underline{\rho}(i)+s, \theta_{M: i}(\underline{r})\right)$ and $\widehat{\beta}_{i}^{*}\left(\underline{r}^{\top} \underline{\rho}(i)+s, \theta_{M: i}(\underline{r})\right)$ at $\underline{r}=\underline{0}$ are given as follows.

$$
\begin{aligned}
\xi_{i j}(s) & =\left.\frac{\partial^{K}}{\partial \underline{r}} \widehat{\beta}_{i j}\left(\underline{r}^{\top} \underline{\rho}(i)+s, \theta_{M: i}(\underline{r})\right)\right|_{\underline{r}=\underline{0}} \\
& =(-1)^{K} \prod_{k=1}^{K} \rho_{k}(i) \int_{0}^{\infty} e^{-s t} t^{K} a_{i j}(t) d t+\Theta_{M: i} \int_{0}^{\infty} e^{-s t} L_{i}(t) a_{i j}(t) d t \\
\xi_{i}^{*}(s) & =\left.\frac{\partial^{K}}{\partial \underline{r}} \widehat{\beta}_{i}^{*}\left(\underline{r}^{\top} \underline{\rho}(i)+s, \theta_{M: i}(\underline{r})\right)\right|_{\underline{r}=\underline{0}} \\
& =(-1)^{K} \prod_{k=1}^{K} \rho_{k}(i) \int_{0}^{\infty} e^{-s t} t^{K} \bar{A}_{i}(t) d t+\Theta_{M: i} \int_{0}^{\infty} e^{-s t} L_{i}(t) \bar{A}_{i}(t) d t
\end{aligned}
$$

Let $\kappa(i)=(-1)^{K} \prod_{k=1}^{K} \rho_{k}(i)$ and define $\underline{\underline{\kappa}}_{D}=\operatorname{diag}\{\kappa(i)\}$. Then Eq.'s (4.2) and (4.3) can be rewritten in matrix form as

$$
\begin{aligned}
& \underline{\underline{\xi}}(s)=\underline{\underline{\kappa}}_{D} \int_{0}^{\infty} e^{-s t} t^{K} \underline{\underline{a}}(t) d t+\underline{\underline{\Theta}}_{M: D} \int_{0}^{\infty} e^{-s t} \underline{\underline{L}}_{D}(t) \underline{\underline{a}}(t) d t \\
& \underline{\underline{\xi}}_{D}^{*}(s)=\underline{\underline{\kappa}}_{D} \int_{0}^{\infty} e^{-s t} t^{K} \underline{\underline{A}}_{D}(t) d t+\underline{\underline{\Theta}}_{M: D} \int_{0}^{\infty} e^{-s t} \underline{\underline{L}}_{D}(t) \underline{\underline{A}}_{D}(t) d t .
\end{aligned}
$$

From Eq. (4.1), after a little algebra, one could see that

$$
\begin{aligned}
\mathscr{L}\left\{\mathrm{E}\left[\prod_{k=1}^{K} Z_{k}(t)\right]\right\} & =\left.(-1)^{K} \frac{\partial^{K}}{\partial \underline{r}} \mathscr{L}\left\{\mathrm{E}\left[e^{-\underline{r}^{\top} \underline{Z}(t)}\right]\right\}\right|_{\underline{r}=\underline{0}} \\
& =(-1)^{K} \underline{\underline{p}}^{\top}(0)\{\underline{\underline{I}}-\underline{\underline{\alpha}}(s)\}^{-1}\left\{\frac{1}{s}\left(\underline{\underline{\Theta}}_{N} \otimes \underline{\underline{\alpha}}(s)+\underline{\underline{\xi}}(s)\right)+\underline{\underline{\xi}}_{D}^{*}(s)\right\} \underline{1} .
\end{aligned}
$$

By taking the Taylor expansion of the Laplace transform $\underline{\underline{\alpha}}(s)$ as $s \rightarrow 0+$, one has

$$
\underline{\underline{\alpha}}(s)=\underline{\underline{A}}_{0}-s \underline{\underline{A}}_{1}+\frac{s^{2}}{2} \underline{\underline{A}}_{2}+\underline{\underline{o}}\left(s^{2}\right)
$$


Let $\underline{\underline{\Phi}}_{d}=\int_{0}^{\infty} t^{d} \underline{\underline{L}}_{D}(t) \underline{\underline{a}}(t) d t$ and $\underline{\underline{\Phi}}_{D: d}^{*}=\int_{0}^{\infty} t^{d} \underline{\underline{L}}_{D}(t) \underline{\underline{A}}_{D}(t) d t, d=0,1,2, \cdots$. By taking the Taylor expansion of $\underline{\underline{\underline{\xi}}}(s)$ and $\underline{\underline{\xi}}_{D}^{*}(s)$, it can be seen that, as $s \rightarrow 0+$,

$$
\begin{aligned}
\underline{\underline{\xi}}(s)= & \left\{\underline{\underline{\kappa}}_{D} \underline{\underline{A}}_{K}+\underline{\underline{\Theta}}_{M: D} \underline{\underline{\Phi}}_{0}\right\}-s\left\{\underline{\underline{\kappa}}_{D} \underline{\underline{A}}_{K+1}+\underline{\underline{\Theta}}_{M: D} \underline{\underline{\Phi}}_{1}\right\}+\frac{s^{2}}{2}\left\{\underline{\underline{\kappa}}_{D} \underline{\underline{A}}_{K+2}+\underline{\underline{\Theta}}_{M: D} \underline{\underline{\Phi}}_{2}\right\}+\underline{\underline{o}}\left(s^{2}\right) \\
\underline{\underline{\xi}}_{D}^{*}(s)= & \left\{\underline{\underline{\kappa}}_{D} \underline{\underline{A}}_{D: K}^{*}+\underline{\underline{\Theta}}_{M: D} \underline{\underline{\Phi}}_{D: 0}^{*}\right\}-s\left\{\underline{\underline{\kappa}}_{D} \underline{\underline{A}}_{D: K+1}^{*}+\underline{\underline{\Theta}}_{M: D} \underline{\underline{\Phi}}_{D: 1}^{*}\right\} \\
& +\frac{s^{2}}{2}\left\{\underline{\underline{\kappa}}_{D} \underline{\underline{A}}_{D: K+2}^{*}+\underline{\underline{\Theta}}_{M: D} \underline{\underline{\Phi}}_{D: 2}^{*}\right\}+\underline{\underline{o}}\left(s^{2}\right) .
\end{aligned}
$$

Of interest is the theorem of Keilson [9] stated as follows.

Theorem 4.1 (Keilson [9]). As $s \rightarrow 0+$, one has

$$
\{\underline{\underline{I}}-\underline{\underline{\alpha}}(s)\}^{-1}=\frac{1}{s} \underline{\underline{H}}_{1}+\underline{\underline{H}}_{0}+\underline{\underline{o}}(1)
$$

where

$$
\begin{aligned}
& \underline{\underline{H}}_{1}=\frac{1}{m} \underline{1}^{\top}, \quad m=\underline{e}^{\top} \underline{\underline{A}}_{1} \underline{1}, \\
& \underline{\underline{H}}_{0}=\underline{\underline{H}}_{1}\left(-\underline{\underline{A}}_{1}+\frac{1}{2} \underline{\underline{A}}_{2} \underline{\underline{H}}_{1}\right)+\left(\underline{\underline{Z}}_{0}-\underline{\underline{H}}_{1} \underline{\underline{A}}_{1} \underline{\underline{Z}}_{0}\right)\left(\underline{\underline{A}}_{0}-\underline{\underline{A}}_{1} \underline{\underline{H}}_{1}\right)+\underline{\underline{I}}^{\prime}, \quad \underline{\underline{Z}}_{0}=\left(\underline{\underline{I}}-\underline{\underline{A}}_{0}+\underline{1} \cdot \underline{e}^{\top}\right)^{-1} .
\end{aligned}
$$

By applying this theorem to Eq. (4.4), and then substituting (4.5), (4.6) and (4.7) into the result, the following theorem holds true.

Theorem 4.2. Let $\underline{p}(0)$ be an initial probability vector of the underlying semi-Markov process $J(t)$. As $t \rightarrow \infty$, one has

$$
E\left[\prod_{k=1}^{K} Z_{k}(t)\right]=(-1)^{K}\left\{\underline{\underline{B}}_{1} \times t+\underline{p}^{\top}(0) \times \underline{\underline{B}}_{0} \times \underline{1}\right\}+o(1),
$$

where

$$
\begin{aligned}
& \left.\underline{\underline{B}}_{1}=\frac{1}{\underline{e}^{\top} \underline{\underline{A}}_{1} \underline{e^{\top}}} \underline{\underline{\Theta}}_{N} \otimes \underline{\underline{A}}_{0}+\underline{\underline{\kappa}}_{D} \underline{\underline{A}}_{K}+\underline{\underline{\Theta}}_{M: D} \underline{\underline{\Phi}}_{0}\right) \underline{1}, \\
& \underline{\underline{B}}_{0}=\underline{\underline{H}}_{0}\left(\underline{\underline{\Theta}}_{N} \otimes \underline{\underline{A}}_{0}+\underline{\underline{\kappa}}_{D} \underline{\underline{A}}_{K}+\underline{\underline{\Theta}}_{M: D} \underline{\underline{\Phi}}_{0}\right)-\underline{\underline{H}}_{1}\left(\underline{\underline{\Theta}}_{N} \otimes \underline{\underline{A}}_{1}+\underline{\underline{\kappa}}_{D}\left(\underline{\underline{A}}_{K+1}-\underline{\underline{A}}_{D: K}^{*}\right)+\underline{\underline{\Theta}}_{M: D}\left(\underline{\underline{\Phi}}_{1}-\underline{\underline{\Phi}}_{D: 0}^{*}\right)\right) .
\end{aligned}
$$

Theorem 4.2 is easy to be extended to the product form of any marginal process of $Z_{k}(t)$ for $k \in \mathscr{H}$ where $\mathscr{H}$ is the arbitrary subset of $\{1, \cdots, K\}$. For notational convenience, the following functions are defined. Let $\underline{\underline{\kappa}}_{D: \mathscr{H}}=\operatorname{diag}\left\{\kappa_{\mathscr{H}}(i)\right\}$ with $\kappa_{\mathscr{H}}(i)=(-1)^{|\mathscr{H}|} \prod_{k \in \mathscr{H}} \rho_{k}(i)$. By considering $\underline{r}_{\mathscr{H}}=\left[\delta_{\{k \in \mathscr{H}\}} r_{k}\right]_{k \in\{1, \cdots, K\}}$, it is shown that

$$
\begin{gathered}
\left.\Theta_{M: i: \mathscr{H}} \stackrel{\text { def }}{=} \frac{\partial^{K}}{\partial \underline{r}_{\mathscr{H}}} \theta_{M: i}\left(\underline{r}_{\mathscr{H}}\right)\right|_{\underline{r}_{\mathscr{H}=0}}=(-1)^{|\mathscr{H}|} \int_{\mathbb{R}^{|\mathscr{H}|}} \prod_{k \in \mathscr{H}} z_{k} \mathscr{Y}_{M: i}(\underline{z}) d \underline{z}, \\
\left.\Theta_{N: i j: \mathscr{H}} \stackrel{\text { def }}{=} \frac{\partial^{K}}{\partial \underline{r}_{\mathscr{H}}} \theta_{N: i j}\left(\underline{r}_{\mathscr{H}}\right)\right|_{\underline{\underline{H}}_{\mathscr{H}}=\underline{0}}=(-1)^{|\mathscr{H}|} \int_{\mathbb{R}^{|\mathscr{H}|}} \prod_{k \in \mathscr{H}} z_{k} \mathscr{Y}_{N: i j}(\underline{z}) d \underline{z},
\end{gathered}
$$


and $\underline{\underline{\Theta}}_{N: \mathscr{H}}=\left[\Theta_{N: i j: \mathscr{H}}\right]_{i, j \in \mathscr{J}}, \underline{\underline{\Theta}}_{M: D: \mathscr{H}}=\operatorname{diag}\left\{\Theta_{M: i: \mathscr{H}}\right\}$.

Corollary 4.3. Let $\mathscr{H}$ be an arbitrary subset of $\{1, \cdots, K\}$ and $Z_{\mathscr{H}}(t)$ be defined as

$$
Z_{\mathscr{H}}(t)=\prod_{k \in \mathscr{H}} Z_{k}(t)
$$

Let $p(0)$ be an initial probability vector of the underlying semi-Markov process $J(t)$. As $t \rightarrow \infty$, one has

$$
E\left[Z_{\mathscr{H}}(t)\right]=(-1)^{|\mathscr{H}|}\left\{\underline{\underline{B}}_{1: \mathscr{H}} \times t+\underline{p}^{\top}(0) \times \underline{\underline{B}}_{0: \mathscr{H}} \times \underline{1}\right\}+o(1)
$$

where

$$
\begin{aligned}
& \underline{\underline{B}}_{1: \mathscr{H}}=\frac{1}{\underline{\underline{e}}^{\top} \underline{\underline{A}}_{1} \underline{\underline{e}}} \underline{ }^{\top}\left(\underline{\underline{\Theta}}_{N: \mathscr{H}} \otimes \underline{\underline{A}}_{0}+\underline{\underline{\kappa}}_{D: \mathscr{H}} \underline{\underline{A}}_{|\mathscr{H}|}+\underline{\underline{\Theta}}_{M: D: \mathscr{H}} \underline{\underline{\Phi}}_{0}\right) \underline{1}, \\
& \underline{\underline{B}}_{0: \mathscr{H}}=\left(\underline{\underline{\Theta}}_{N: \mathscr{H}} \otimes \underline{\underline{A}}_{0}+\underline{\underline{\kappa}}_{D: \mathscr{H}} \underline{\underline{A}}_{|\mathscr{H}|}+\underline{\underline{\Theta}}_{M: D: \mathscr{H}} \underline{\underline{\Phi}}_{0}\right) \\
& -\underline{\underline{H}}_{1}\left(\underline{\underline{\Theta}}_{N: \mathscr{H}} \otimes \underline{\underline{A}}_{1}+\underline{\underline{\kappa}}_{D: \mathscr{H}}\left(\underline{\underline{A}}_{|\mathscr{H}|+1}-\underline{\underline{A}}_{D:|\mathscr{H}|}^{*}\right)+\underline{\underline{\Theta}}_{M: D: \mathscr{H}}\left(\underline{\underline{\Phi}}_{1}-\underline{\underline{\Phi}}_{D: 0}^{*}\right)\right) \text {. }
\end{aligned}
$$

The proof of Corollary 4.3 is similar to that of Theorem 4.2 and is omitted here.

In the next section, Theorem 4.2 is applied to the optimal preventive maintenance policy problem with state dependent continuous revenues and costs, as well as random cost increments at the times of jumps of $M_{i}(t)$ and $N_{i j}(t)$.

\section{Optimal Preventive Maintenance Policy Problem}

We consider a manufacturing system with a certain maintenance policy, where the system starts with the perfect state at time $t=0$, and tends to degrade, generating product defects more often, as time goes by. When the system reaches a certain state, the manufacturing system would be overhauled completely and the system returns to the perfect state. More specifically, let $J(t)$ be a semi-Markov process on $\mathscr{J}=\{0,1,2, \cdots, J\}$ governed by $\underline{\underline{A}}(x)$, where $x$ denotes the dwell time of the process in the current state, that is, the elapsed time since the last transition into the current state. Here, the semiMarkov process $J(t)$ describes the system state at time $t$ where state 0 is the perfect state and state $J$ is the maintenance state. When the system is in state $j, 1 \leq j \leq J-1$, product defects are generated according to an NHPP with intensity $\lambda_{j}(x)$. It is assumed that the system deteriorates monotonically and accordingly $\lambda_{j}(x)$ increases as a function of both $x$ and $j$. When the system reaches state $J$, the manufacturing operation is stopped and the system is overhauled completely. The maintenance time increases stochastically as a function of $J$. In other words, the further the maintenance is delayed, the longer the maintenance time would tend to be. Upon finishing the overhaul, the system is brought back to the perfect state 0 . The state transitions of this system are depicted in Figure 2. Of interest, then, is to determine the optimal preventive maintenance policy concerning how to set $J$.

In order to determine the optimal preventive maintenance policy, it is necessary to define the objective function precisely. In Sumita and Huang [26], a function capturing the total cost generated by the system is employed. However, the cost structure is limited in that all the costs are assumed to be constant and are incurred only at the times of jumps of $M_{i}(t)$ and $N_{i j}(t)$. Furthermore, the revenue side is totally ignored. In what follows, these pitfalls are overcome by applying the multivariate 


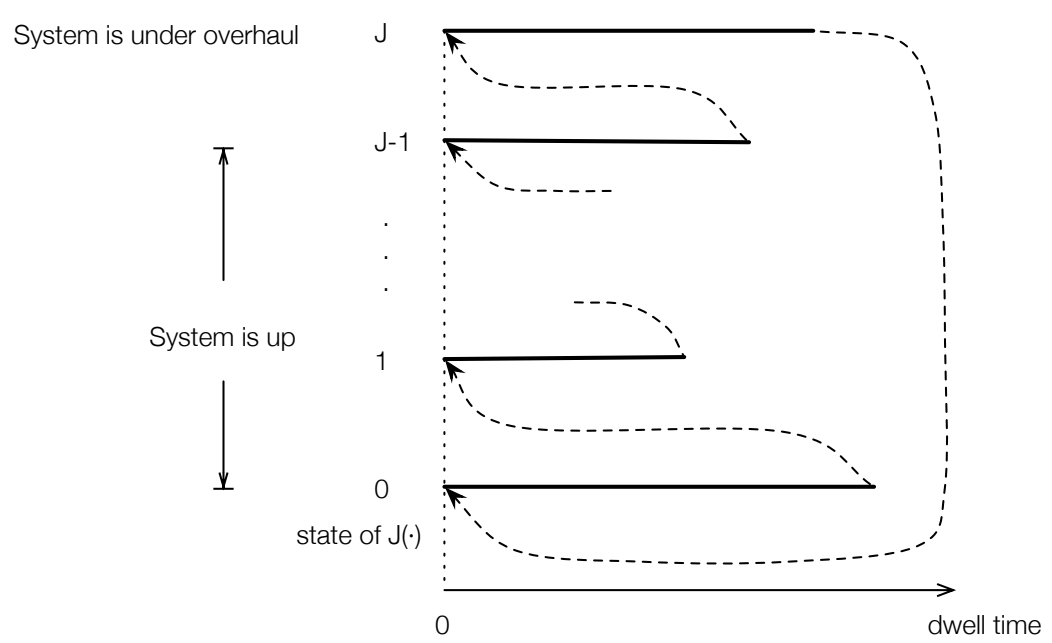

Figure 2: State Transitions of the Manufacturing System

reward process discussed in Section 3 to the optimal preventive maintenance policy problem, where the revenue-cost structure in continuous time is now incorporated. More formally, let $\rho: \mathscr{J} \rightarrow \mathbb{R}$ be the revenue-cost function defined by

$$
\rho(i)=\left\{\begin{array}{ll}
\rho_{\text {rev }}>0 & \text { for } i=0, \cdots, J-1 \\
-\rho_{\text {cost }}<0 & \text { for } i=J
\end{array} .\right.
$$

Here, the manufacturing system generates the revenue $\rho_{\text {rev }}$ per unit time whenever the system is up, and the cost of $\rho_{\text {cost }}$ per unit time is incurred when the system is under the overhaul.

Let $Z(t)$ be the cumulative profit up to time $t$, which is univariate. $M_{i}(t)$ and $N_{J-1, J}(t)$ denote the total number of defects generated by time $t$ while $J(t)=i$ and the number of the maintenance operations occurred by time $t$ respectively. One then sees that

$$
Z(t)=\int_{0}^{t} \rho(J(t)) d t+\sum_{i=0}^{J-1} \sum_{d=1}^{M_{i}(t)} Y_{M: i: d}+\sum_{d=1}^{N_{J-1, J}} Y_{N: J-1, J: d},
$$

where $Y_{M: i: d}$ represents the cost for each defect and is naturally assumed to be constant, i.e. $Y_{M: i: d}=$ $-\psi_{d}<0$ for $i=0, \cdots, J-1 . Y_{N: J-1, J: d}$ describes the cost associated with each occurrence of the overhaul and may not be constant. For example, the overhaul may or may not require the presence of engineer(s) from the vender of the production machines. Hence, we assume that $Y_{N: J-1, J: d}$ constitute a sequence of i.i.d. random variables with respect to $d$ having the common expected value $-\psi_{m}<0$. It is worth noting that the subscripts in $\psi_{d}$ and $\psi_{m}$ stand for "defect" and "maintenance", respectively.

The problem now is to determine the optimal preventive maintenance policy $J^{*}$ so as to maximize the expected profit, that is,

$$
\pi_{J^{*}}(T)=\max _{J \in \mathbb{N}} \pi_{J}(T) ; \quad \pi_{J}(T)=\mathrm{E}[Z(T)]
$$

When $T$ is reasonably large, the asymptotic result of Theorem 4.2 can be employed so as to solve the maximization problem of (5.1) approximately. Since the reward process in the above application 
is univariate, one has $K=1$ in Theorem 4.2 and the matrices involved in the asymptotic expansion can be rewritten accordingly, e.g. $\underline{\underline{\kappa}}_{D}=-\operatorname{diag}\{\rho(i)\}$, etc. In the next section, we provide numerical examples, demonstrating efficiency of the proposed approach.

\section{Numerical Results}

In order to provide numerical examples for the optimal preventive maintenance policy problem introduced in the previous section, we consider the semi-Markov matrix $\underline{\underline{A}}(x)$ of the form

$$
\underline{\underline{A}}(x)=\left[\begin{array}{ccccc}
0 & A_{1}(x) & 0 & \cdots & 0 \\
0 & 0 & A_{2}(x) & 0 & \vdots \\
\vdots & \vdots & \ddots & \ddots & 0 \\
0 & 0 & \cdots & 0 & A_{J-1}(x) \\
A_{J}(x) & 0 & \cdots & \cdots & 0
\end{array}\right] .
$$

We recall that the manufacturing system deteriorates gradually from state 0 to state $J-1$ and the overhaul activity takes place in state $J$. This point is reflected by setting the expected dwell time in state $i$ to be $\mu_{i}=i+1, i=0, \cdots, J$. In order to specify $A_{i}(x)$ further, we employ gamma distributions given by

$$
a_{i}(x)=\frac{\beta_{i}}{\Gamma\left(\alpha_{i}\right)}\left(\beta_{i} x\right)^{\alpha_{i}-1} e^{-\beta_{i} x} .
$$

Two different cases are considered: the IFR (increasing failure rate) case and the DFR (decreasing failure rate) case. For the former, we set $\alpha_{i}=\alpha_{I F R}=2, i=0, \cdots, J-1$, and $\alpha_{J}=1$, while for the latter one has $\alpha_{i}=\alpha_{D F R}=0.2, i=0, \cdots, J-1$, and $\alpha_{J}=1$. $\beta_{i}$ 's are given so as to have $\mu_{i}$ as the expectation, i.e. $\beta_{i}=\alpha_{i} / \mu_{i}, i=0, \cdots, J$. The arrival intensity function in state $i$ is given by $\lambda_{i}(x)=2 i x$. Other parameters are set to be $\rho_{\text {rev }}=1500, \rho_{\text {cost }}=1000, \psi_{d}=10, \psi_{m}=2000+200 \sqrt{J}$ and $T=1000$.

In addition to the expected profit $\pi_{J}(T)$, two availability measures of interest are also evaluated. For this purpose, we define

$$
U(t)=\int_{0}^{t} \rho_{u}(J(t)) d t \text { where } \rho_{u}(i)=\left\{\begin{array}{ll}
1 & i \in\{0, \cdots, J-1\} \\
0 & i=J
\end{array} .\right.
$$

Two traditional measures can now be described as

$$
\operatorname{MTBF}_{J}(t)=\frac{\mathrm{E}[U(t)]}{\mathrm{E}\left[N_{J-1, J}(t)\right]} ; \quad \operatorname{AVAIL}_{J}(t)=\frac{\mathrm{E}[U(t)]}{t} .
$$

Here, MTBF stands for the mean time between failures where a failure means an overhaul in our model. AVAIL describes the average availability per unit time.

Figures 3 through 5 illustrate the asymptotic behaviors of $\pi_{J}(T), \operatorname{MTBF}_{J}(T)$ and $\operatorname{AVAIL}_{J}(T)$ as a function of $J$ respectively, where four different curves correspond to $T=25,50,100$ and 1000 . We first note that our model is reduced to an alternating renewal process with $J=1$. In this case, with $\rho_{\text {rev }}=1500$ and $\rho_{\text {cost }}=1000$, one may expect that the cost for overhauling the system would overwhelm the revenue from the production. This phenomenon can be observed in Figure 3 where $\pi_{1}(T)<0$ for all $T=25,50,100$ and 1000 for all of the IFR, CFR and DFR cases. Furthermore, 
when $J=1, T=25$ is long enough to reach the ergodicity and $\pi_{1}(T)$ 's are almost all equal for $T=25,50,100$ and 1000 . As $J$ increases, it takes more time to reach the ergodicity and the discrepancy among the values of $\pi_{J}(T)$ for different values of $T$ becomes larger. For each fixed $J, \pi_{J}(T)$ decreases as $T$ increases. When $T=25, \pi_{J}(T)$ increases as $J$ increases for all of the IFR, CFR and DFR cases, having the optimal preventive maintenance policy $J^{*}=10$. As $T$ increases, however, the concavity sets in and the optimal preventive maintenance policy changes as depicted in Table 1. For the IFR case, one sees that $J^{*}=7$ with $T=1000$, while the corresponding optimal preventive maintenance policy is $J^{*}=6$ for the CFR case and $J^{*}=4$ for the DFR case. This demonstrates the potential danger of the exclusive reliance on ergodicity. In Figures 4 and 5, we observe that both $\operatorname{MTBF}_{J}(T)$ and $\operatorname{AVAIL}_{J}(T)$ increase as $J$ increases, and decrease as $T$ increases. One sees that it could be misleading to design the optimal preventive maintenance strategy based on the availability measures alone, highlighting the importance to incorporate the reward process.

Table 1: Optimal Preventive Maintenance Policy $J^{*}$

\begin{tabular}{lcccc}
\hline & $T=25$ & $T=50$ & $T=100$ & $T=1000$ \\
\hline IFR & 10 & 8 & 7 & 7 \\
CFR & 10 & 8 & 7 & 6 \\
DFR & 10 & 10 & 5 & 4 \\
\hline
\end{tabular}

\section{Acknowledgement}

The authors wish to thank an anonymous referee and the editor for providing constructive comments, which contributed to improve the original version of the paper substantially. This research is supported by MEXT Grant-in-Aid for Scientific Research (C) 22500082.

\section{References}

1. Barlow, R. \& Hunter, L. (1960). Optimum Preventive Maintenance Policies. Operations Research 8: $90-100$.

2. Çınlar, E. (1969). Markov Renewal Theory. Advances in Applied Probability 1: 123-187.

3. Çınlar, E. (1975). Markov Renewal Theory: A Survey. Management Science 21: 727-752.

4. Feller, W. (1950). An introduction to probability theory and its applications, vol. 1. Wiley.

5. Flehinger, B. J. (1962). A General Model for the Reliability Analysis of Systems Under Various Preventive Maintenance Policies. The Annals of Mathematical Statistics 33: 137-156.

6. Heffes, H. \& Lucantoni, D. M. (1986). A Markov Modulated Characterization of Packetized Voice and Data Traffic and Related Statistical Multiplexer Performance. IEEE Journal on Selected Areas in Communications 4: 856-868. 


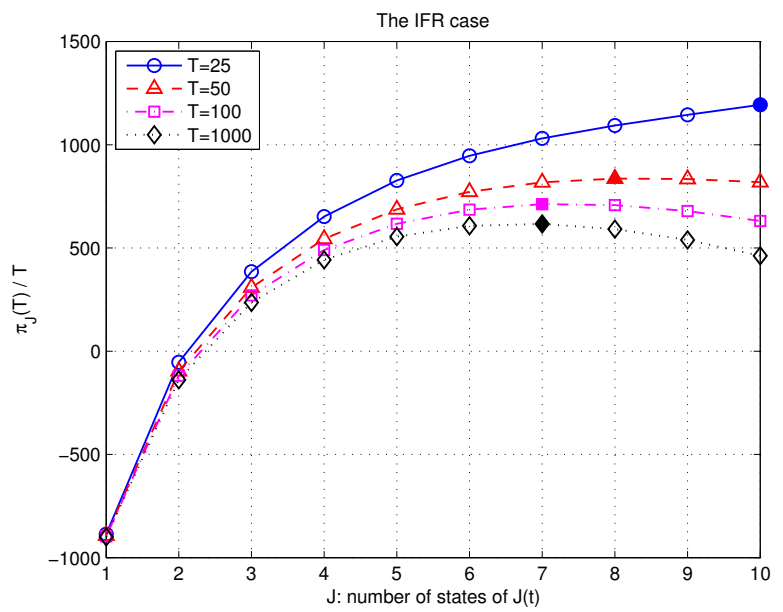

(a)

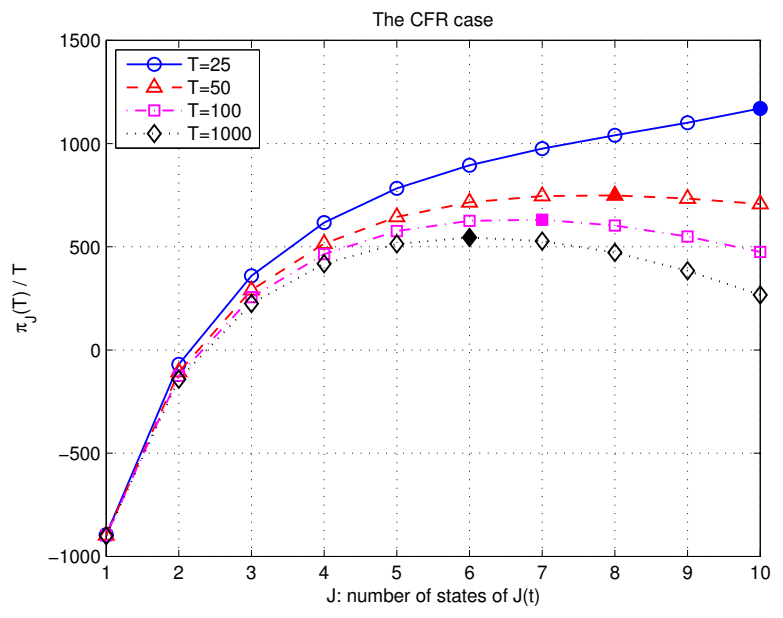

(b)

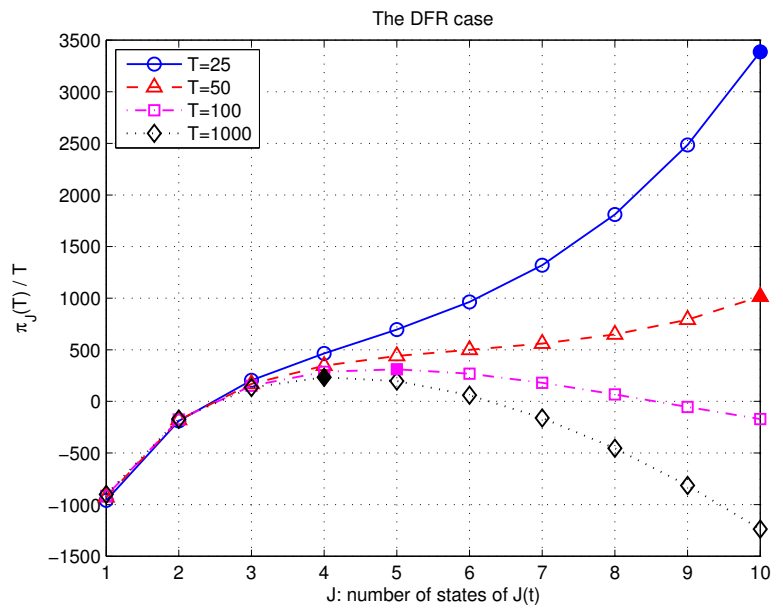

(c)

Figure 3: $\pi_{J}(T) / T$ as a function of $J$ 


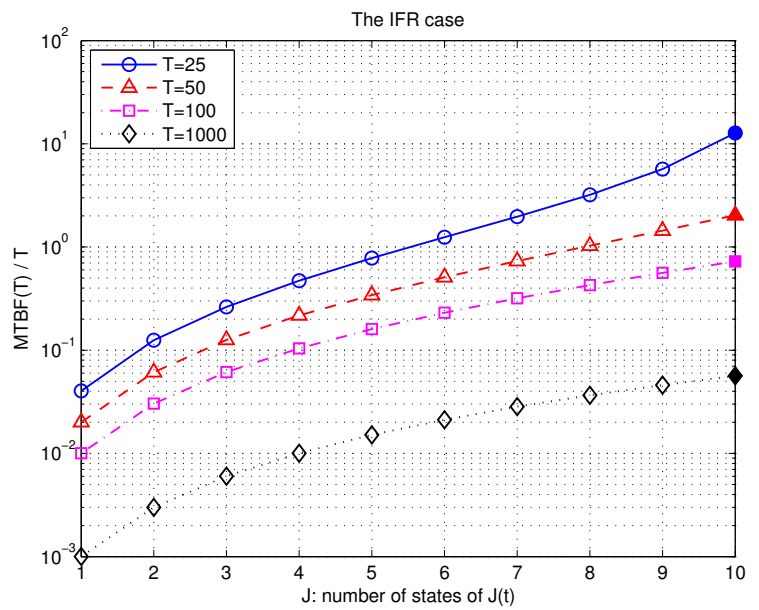

(a)

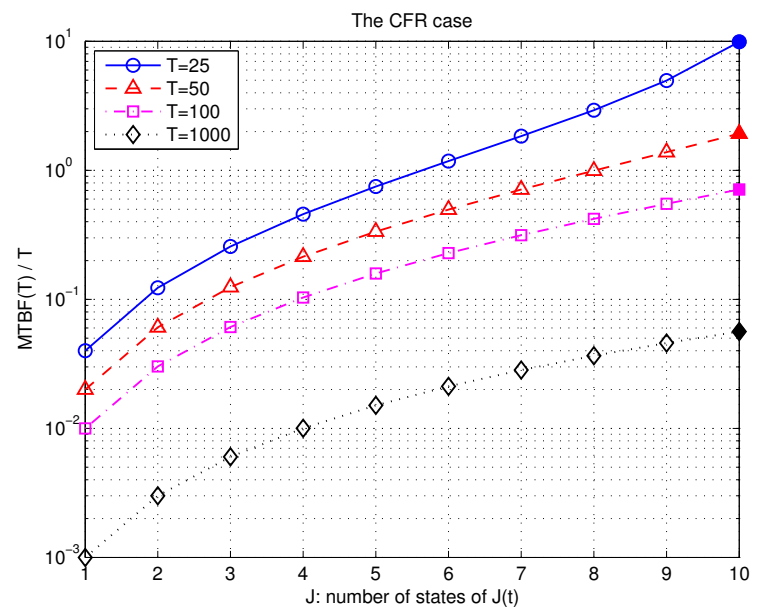

(b)

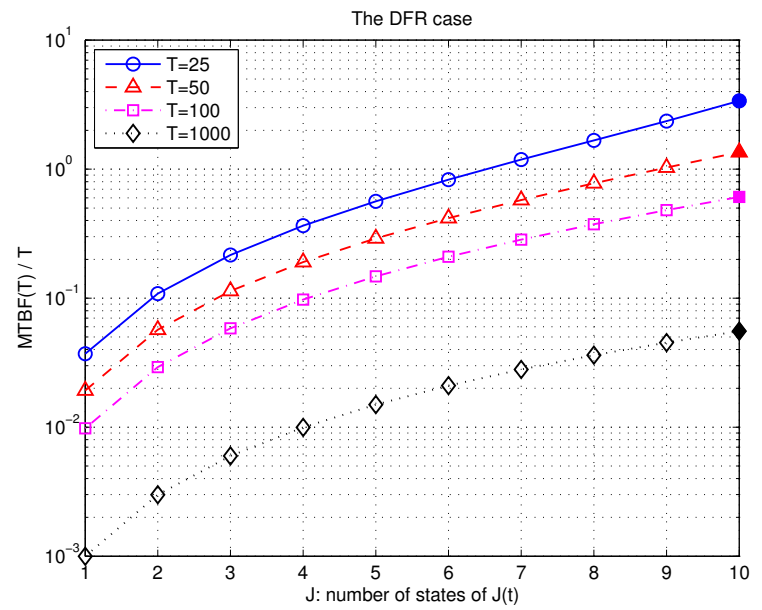

(c)

Figure 4: $\log$-scaled $\operatorname{MTBF}(T) / T$ as a function of $J$ 


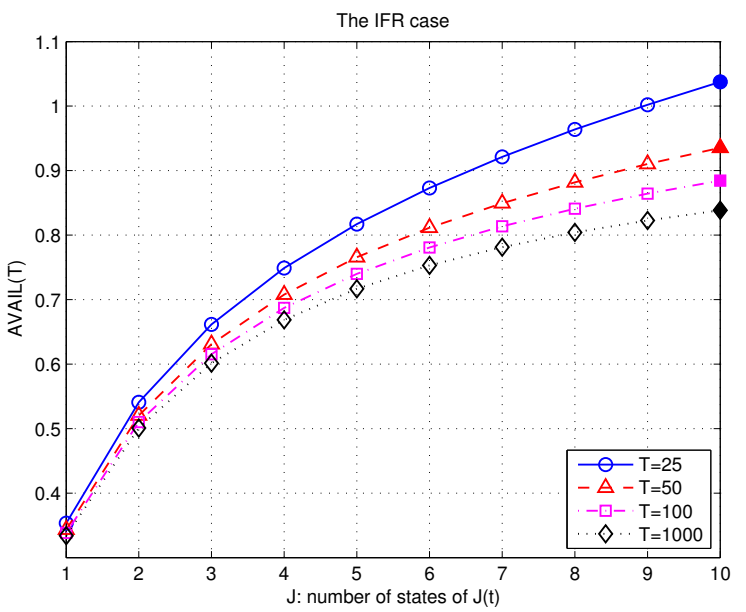

(a)

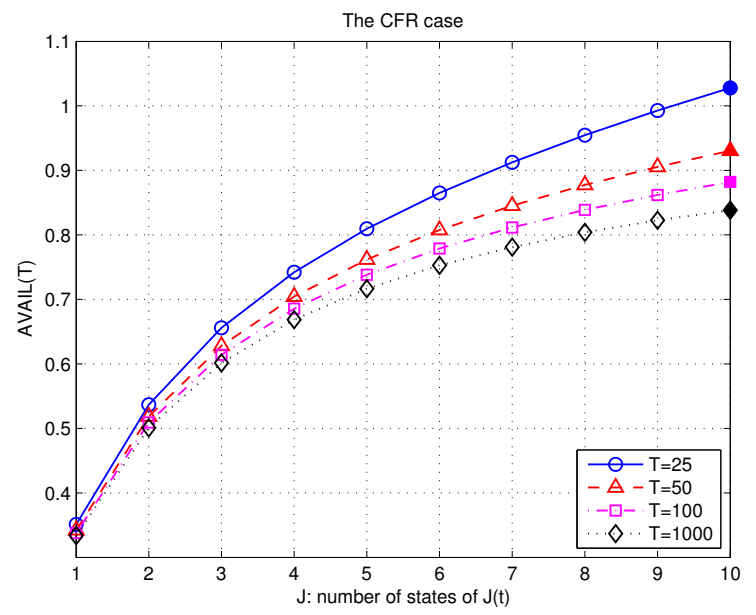

(b)

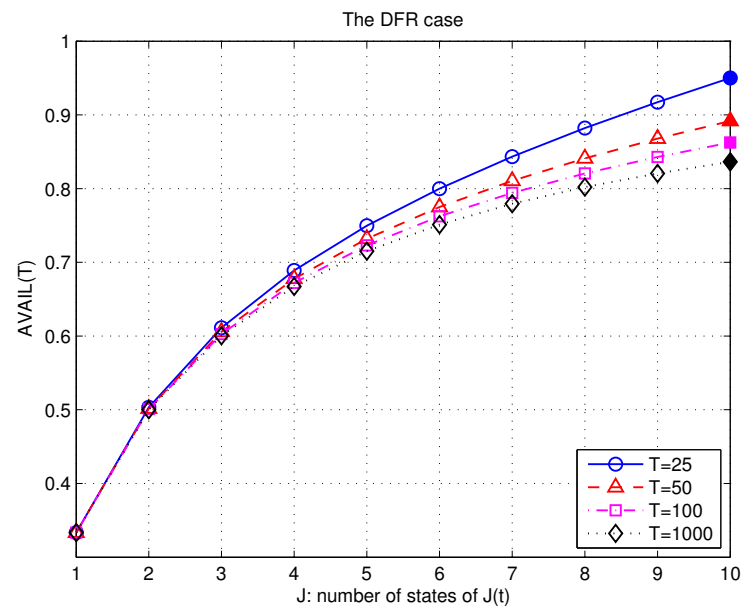

(c)

Figure 5: $\operatorname{AVAIL}(T)$ as a function of $J$ 
7. Jewell, W. S. (1963). Markov-Renewal Programming. I: Formulation, Finite Return Models. Operations Research 11: 938 - 948.

8. Keilson, J. (1966). A Limit Theorem for Passage Times in Ergodic Regenerative Processes. The Annals of Mathematical Statistics 37: 866-870.

9. Keilson, J. (1969). On the Matrix Renewal Function for Markov Renewal Processes. The Annals of Mathematical Statistics 40: 1901-1907.

10. Keilson, J. \& Wishart, D. M. G. (1964). A central limit theorem for processes defined on a finite Markov. Proceedings of the Cambridge Philosophical Society 60: 547-567.

11. Keilson, J. \& Wishart, D. M. G. (1965). Boundary problems for additive processes defined on a finite markov chain(Homogeneous additive process defined on Markov chain with boundary states modification, noting Green function and passage time density). Proceedings of the Cambridge Philosophical Society 61: 173-190.

12. Lucantoni, D. M. (1991). New Results on the Single Server Queue with a Batch Markovian Arrival Process. Stochastic Models 7: 1-46.

13. Lucantoni, D. M., Meier-Hellstern, K. S. \& Neuts, M. F. (1990). A Single Server Queue with Server Vacations and a Class of Non-Renewal Arrival Processes. Advances in Applied Probability 22: $676-705$.

14. Malik, M. A. K. (1979). Reliable Preventive Maintenance Scheduling. A I I E Transactions 11: 221-228.

15. Masuda, Y. (1993). Partially Observable Semi-Markov Reward Processes. Journal of Applied Probability 30: 548-560.

16. Masuda, Y. \& Sumita, U. (1987). Analysis of a Counting Process Associated with a Semi-Markov Process: Number of Entries into a Subset of State Space. Advances in Applied Probability 19: 767-783.

17. Masuda, Y. \& Sumita, U. (1991). A Multivariate Reward Process Defined on a Semi-Markov Process and Its First-Passage-Time Distributions. Journal of Applied Probability 28: 360-373.

18. McLean, R. A. \& Neuts, M. F. (1967). The Integral of a Step Function Defined on a Semi-Markov Process. SIAM Journal on Applied Mathematics 15: 726-737.

19. Pierskalla, W. P. \& Voelker, J. A. (1976). A survey of maintenance models: The control and surveillance of deteriorating systems. Naval Research Logistics Quarterly 23: 353-388.

20. Pyke, R. (1961). Markov Renewal Processes: Definitions and Preliminary Properties. The Annals of Mathematical Statistics 32: 1231-1242.

21. Pyke, R. (1961). Markov Renewal Processes with Finitely many states. The Annals of Mathematical Statistics 32: 1243-1259.

22. Pyke, R. \& Schaufele, R. (1964). Limit Theorems for Markov Renewal Processes. The Annals of Mathematical Statistics 35: 1746-1764. 
23. Ross, S. M. (1996). Stochastic processes. Wiley, 2ed edition.

24. Smith, W. L. (1955). Regenerative Stochastic Processes. Proceedings of the Royal Society of London. Series A, Mathematical and Physical Sciences 232: 6 - 13.

25. Stefanov, V. T. (2006). Exact Distributions for Reward Functions on Semi-Markov and Markov Additive Processes. Journal of Applied Probability 43: 1053 - 1065.

26. Sumita, U. \& Huang, J.-P. (2009). Dynamic Analysis of a Unified Multivariate Counting Process and Its Asymptotic Behavior. International Journal of Mathematics and Mathematical Sciences vol.2009: Article ID 219532, 43pages. Doi:10.1155/2009/219532.

27. Sumita, U. \& Masuda, Y. (1987). An Alternative Approach to the Analysis of Finite Semi-Markov and Related Processes. Stochastic Models 3: 67-87.

28. Sumita, U. \& Shanthikumar, J. G. (1988). An Age-Dependent Counting Process Generated from a Renewal Process. Advances in Applied Probability 20: 739-755.

29. Valdez-Flores, C. \& Feldman, R. M. (1989). A survey of preventive maintenance models for stochastically deteriorating single-unit systems. Naval Research Logistics 36: 419-446.

30. Wang, H. (2002). A survey of maintenance policies of deteriorating systems. European Journal of Operational Research 139: 469-489. 